\title{
Efficient Bayesian uncertainty estimation in linear finite fault inversion with positivity constraints by employing a log-normal prior
}

\author{
Roberto Benavente ${ }^{\odot}, 1,2$ Jan Dettmer, ${ }^{3}$ Phil R. Cummins ${ }^{4}$ and Malcolm Sambridge ${ }^{4}$ \\ ${ }^{1}$ National Research Center for Integrated Natural Disaster Management (CIGIDEN), Santiago, Chile. E-mail: rbenavente@ucsc.cl \\ ${ }^{2}$ Department of Civil Engineering, Universidad Católica de la Santísima Concepción, Concepción, Chile \\ ${ }^{3}$ Department of Geosciences, University of Calgary, Calgary AB T2N 1N4, Canada \\ ${ }^{4}$ Research School of Earth Sciences, Australian National University, Canberra ACT 2601, Australia
}

Accepted 2019 January 22. Received 2019 January 19; in original form 2018 August 27

\begin{abstract}
SUMMAR Y
Obtaining slip distributions for earthquakes results in an ill-posed inverse problem. While this implies that only limited and uncertain information can be recovered from the data, inferences are typically made based only on a single regularized model. Here, we develop an inversion approach that can quantify uncertainties in a Bayesian probabilistic framework for the finite fault inversion (FFI) problem. The approach is suitably efficient for rapid source characterization and includes positivity constraints for model parameters, a common practice in FFI, via coordinate transformation to logarithmic space. The resulting inverse problem is nonlinear and the most probable solution can be obtained by iterative linearization. In addition, model uncertainties are quantified by approximating the posterior probability distribution by a Gaussian distribution in logarithmic space. This procedure is straightforward since an analytic expression for the Hessian of the objective function is obtained. In addition to positivity, we apply smoothness regularization to the model in logarithmic space. Simulations based on surface wave data show that smoothing in logarithmic space penalizes abrupt slip changes less than smoothing in linear space. Even so, the main slip features of models that are smooth in linear space are recovered well with logarithmic smoothing. Our synthetic experiments also show that, for the data set we consider, uncertainty is low at the shallow portion of the fault and increases with depth. In addition, a simulation with a large station azimuthal gap of $180^{\circ}$ significantly increases the slip uncertainties. Further, the marginal posterior probabilities obtained from our approximate method are compared with numerical Markov Chain Monte Carlo sampling. We conclude that the Gaussian approximation is reasonable and meaningful inferences can be obtained from it. Finally, we apply the new approach to observed surface wave records from the great Illapel earthquake (Chile, 2015, $M_{\mathrm{w}}=8.3$ ). The location and amplitude of our inferred peak slip is consistent with other published solutions but the spatial slip distribution is more compact, likely because of the logarithmic regularization. We also find a minor slip patch downdip, mainly in an oblique direction, which is poorly resolved compared to the main slip patch and may be an artefact. We conclude that quantifying uncertainties of finite slip models is crucial for their meaningful interpretation, and therefore rapid uncertainty quantification can be critical if such models are to be used for emergency response.
\end{abstract}

Key words: Inverse theory; Probability distributions; Earthquake source observations.

\section{INTRODUCTION}

Seismic source studies rely on incomplete spatial sampling and noisy measurements of the displacement field at the Earth's surface. Nonetheless, by using these data, we can still study the spatiotemporal evolution of earthquake rupture (Olson \& Apsel 1982). Similar to many other geophysical inverse problems, the incomplete and noisy observations lead to uncertain knowledge about the inferred model. This non-uniqueness of the solution means that many models can fit the observed data reasonably well, although some of those models may not be plausible. To avoid implausible models, a common remedy is to include additional constraints on the solution. Typical examples in Finite Fault Inversion (FFI) are Tikhonov regularization (e.g. Tikhonov \& Arsenin 1977) and positivity 
constraints. The former involves the introduction of often subjective prior information about the rupture pattern that needs to be adjusted such that the final solution still fits the data. The latter limits the possible slip directions on the fault. Once these conditions have been adjusted to the particular problem of interest, a single optimal solution can often be selected as the most plausible that explains the data to a reasonable extent but that exhibits some bias due to regularization. However, to better understand the information observed data provide about rupture, it is desirable to consider not only optimal models but all models that sufficiently explain the data, a concept referred to as uncertainty estimation.

An approach that quantifies uncertainty is given by Bayesian probability: Unknown parameters are considered random variables and prior knowledge about these parameters is updated with information from observations to gain insight into the range of solutions that explain the data. Importantly, this approach explicitly acknowledges that model inferences are limited by noise on the data and probabilities express degrees of belief (Tarantola \& Valette 1982). The information is in terms of such probabilities and the solution to the problem is given by a probability density function (PDF) known as a posterior PDF: The state of information about the parameters given prior and data information. From the posterior, we can extract various parameter estimates such as the most probable parameter values and parameter uncertainties. Early Bayesian inference studies focus on the linear regression problem, stressing the advantages of the use of prior information and exploiting posterior PDF properties to obtain robust statistical solutions, compared with the standard optimization approach (e.g. Lindley \& El-Sayyad 1968; Lindley \& Smith 1972; Smith 1973; Box \& Tiao 1968). Schwarz et al. (1978) devised a method to estimate the number of model parameters by using a specific class of priors. This technique, termed Bayesian Information Criterion (BIC), is well known today and can be seen as a Bayesian alternative to the maximum likelihood based technique previously derived for the same purpose by Akaike (1974).

The Bayesian probabilistic framework has been employed previously in FFI, addressing different levels of complexity in the inverse problem. Early applications (e.g. Yoshida 1989; Yabuki \& Matsu'ura 1992; Ide et al. 1996) posed the inverse problem probabilistically and solved for the maximum a posteriori (MAP) model using conventional least-squares techniques. These works applied Akaike's BIC (ABIC; Akaike 1980) to objectively estimate optimal values for the rupture complexity (e.g. regularization by smoothness) and the noise level on the data (i.e. the noise standard deviation). However, even after several decades of application, the procedures to select regularization and noise parameters are still poorly discussed in many other FFI studies.

When the FFI is formulated as linear (no positivity constraints and a linear source time function), it is possible to write an analytic expression for the posterior PDF which, in this special case, is a multivariate Gaussian (Fukuda \& Johnson 2008), assuming Gaussian likelihood and prior. In addition, the MAP model can be found directly from linear least-squares inversion. These analytic solutions can be exploited to efficiently explore, within a probabilistic framework, dependence of the solution on nonlinear model assumptions such as relative weights of different data sets and geometry of the faulting plane (e.g. Fukuda \& Johnson 2010; Minson et al. 2014a). In these cases, the use of analytic expressions for the linearized part of the problem results in a more computationally tractable problem since direct sampling is only employed for some parameters.

Application of Bayesian methods to nonlinear inverse problems typically involves Markov Chain Monte Carlo (MCMC) sampling, that is, the posterior PDF is pointwise evaluated with large numbers of dependent samples of the parameter vector from a Markov chain. Note that MCMC sampling methods provide a natural way to estimate regularization parameters by including them as unknowns in the problem (Fukuda \& Johnson 2008). With increasing computer power, MCMC sampling methods are increasingly utilized in FFI (e.g. Fukuda \& Johnson 2008; Monelli \& Mai 2008; Dettmer et al. 2014; Minson et al. 2014b; Duputel et al. 2015; Kubo et al. 2016). While MCMC sampling is general and does not rely on linearization to estimate the posterior PDF, it is computationally demanding due to the requirement to evaluate the forward problem for extremely large numbers of candidate models.

Therefore, a common way of parametrizing FFI of waveform data is to linearize the problem (e.g. Olson \& Apsel 1982; Hartzell \& Heaton 1983; Hartzell et al. 1996; Ide 2007). The linearization is achieved by fixing the fault geometry and discretizing the fault surface as subfaults whose source time functions are approximated by linear combinations of simple functions (e.g. triangles). Each function represents a time window in which the subfault may exhibit slip. The approach is referred to as the multiple time window method (MTWM); it is linear and can account for some variability in rupture velocity and shape of the source time function. A disadvantage is the requirement for large numbers of parameters, since each subfault requires slip parameters for each time window and many time windows are often applied. On the other hand, explicitly solving for the rupture velocity or subfault rise-time requires nonlinear optimization (e.g. Ji et al. 2002).

Assuming that the linear approximation is reasonable, the posterior PDF is given analytically by a Gaussian PDF (e.g. Tarantola 2005; Fukuda \& Johnson 2008), assuming Gaussian likelihood and prior, and can be estimated efficiently. However, positivity constraints are often employed as additional regularization to obtain plausible inferences. Positivity constraints restrict the solution to only allow slip in certain directions and avoid unlikely, opposing slip directions across the fault. Consequently, under positivity constraints the prior cannot be reasonably represented by a Gaussian, which has support over all real numbers. Fukuda \& Johnson (2008) show that, when a truncated Gaussian is employed as a prior, to achieve positivity, the posterior is no longer Gaussian and employs MCMC sampling to find it. For this case Nocquet (2018) shows that the posterior is a truncated Gaussian; however, parameter marginals are not Gaussian nor Truncated Gaussian (Cartinhour 1990).

Therefore, integrals of the posterior are not trivial and no analytic expression exists for the posterior covariance matrix. Such analytic expressions are required, for example, to apply the ABIC and to infer slip uncertainties, even though the most probable solution can still be found by employing the non-negative least-squares algorithm (NNLS; Lawson \& Hanson 1974). In the absence of analytic expressions for the ABIC, MCMC sampling can be applied to objectively constrain noise and smoothing parameters when positivity constraints are applied (Fukuda \& Johnson 2008; Kubo et al. 2016). In that MCMC sampling approach, positivity constraints are implemented by bounded priors that restrict the parameter space of the inversion. Moreover, integrals of the posterior PDF are evaluated numerically and do not require analytic solutions, account for uncertainty of the smoothing parameters, and are less likely to exhibit linearization error. However, computational cost is much higher and such methods are therefore unsuitable for time-sensitive application.

In recent work, Nocquet (2018) shows that a truncated Gaussian prior can be applied and relevant posterior statistics (e.g. parameter 
marginals, mean and covariances) can be well approximated without performing MCMC sampling. The approach employs recent findings in truncated Gaussian statistics (Genz \& Bretz 2009). The multidimensional integral required to obtain parameter marginals is efficiently evaluated by a numerical algorithm, after a coordinate transformation to a more suitable integration domain. Nocquet (2018) goes on to show that this approach can incorporate additional upper bounds on fault slip and be extended to include a mixture of parameters with and without positivity constraints. The method is applied to the modelling of interseismic crustal deformation of central Peru from GPS data.

In this work, we show an alternative approach to slip uncertainty quantification under positivity constraints that does not rely on MCMC sampling and is based on an untruncated prior, the lognormal distribution. We apply a parameter transformation to the scalar moment (or, equivalently, slip) of each subfault and invert for the natural logarithm of the parameters. This procedure is equivalent to assume a multivariate log-normal prior for scalar moment and, therefore, differs from truncated Gaussian prior previously discussed. The transformation ensures positivity and the posterior can be approximated by a Gaussian distribution in logarithmic space. The solution in linear space around the MAP solution is given by a log-normal PDF from which we can obtain analytic expressions for marginal distributions to quantify uncertainties. In this article, we first develop the appropriate Bayesian framework to be used in the inversion scheme. Particular attention is given to the computation of uncertainties from the posterior PDF. Next, we discuss the problem of estimating the MAP solution, which is a nonlinear optimization problem. Then, we apply our method to simulated surface wave data that are modelled to mimic the Illapel event $\left(M_{\mathrm{w}}=8.3,2015\right.$, Chile), studying the effects of the smoothing in logarithmic space and the azimuthal station coverage. The simulation results are compared to MCMC sampling estimates. This systematic comparison provides some insight into the linearization errors due to the posterior approximation. Finally, the method is applied to surface wave observations from the Illapel earthquake. We compute uncertainties for the cumulative slip distribution for 2-D marginals of each rake component as well as the slip magnitude. Comparison to an MCMC sampling is also offered.

\section{BAYESIAN INVERSION FRAMEWORK}

This section presents a Bayesian framework for MTWM FFI. We begin with the formulations of Fukuda \& Johnson (2008) and Kubo et al. (2016), and then introduce a variable transformation to obtain an approximated posterior that does not require an MCMC sampling. In Bayesian inference, we seek the posterior PDF from the Bayes' theorem (e.g. Tarantola 2005; Sivia \& Skilling 2006). For $N$ data $\mathbf{d}$ and $M$ parameters $\mathbf{m}$, the posterior PDF $P(\mathbf{m} \mid \mathbf{d})$ is given by

$P(\mathbf{m} \mid \mathbf{d})=C P(\mathbf{d} \mid \mathbf{m}) P(\mathbf{m})$,

where $C$ is a normalization constant and $P(\mathbf{m})$ is the prior, expressing knowledge about the parameters that is independent from the data. For observed data, the data error distribution $P(\mathbf{d} \mid \mathbf{m})$ is interpreted as a likelihood function (a function of $\mathbf{m}$ ) that quantifies the likelihood that the model parameters gave rise to the data.

\subsection{Parametrization and positivity constraints}

In FFI we seek to infer the spatial and temporal evolution of fault rupture from ground motions detected at the surface. The problem is parametrized by spatial and temporal discretization: The fault surface is assumed to be known and discretized with subfaults. It is common to represent slip by two orthogonal vectors of prescribed rake angles with unknown magnitude to incorporate unknown rake in the inversion. The temporal evolution can be parametrized as nonlinear or linearized. A common linearized method is MTWM that can account for some variability in the temporal evolution of rupture while allowing linear methods to be applied (e.g. Ide 2007). Here, we apply MTWM discretization to achieve computational efficiency.

In MTWM, $N_{\mathrm{tw}}$ rupture fronts propagate with a prescribed rupture velocity $v_{\mathrm{r}}^{\max }$, separated by time interval $t_{\mathrm{d}}$. A particular subfault can slip each time a rupture front reaches it and the temporal dependence of each slip event follows the specified source time function, typically a triangle function of half duration $t_{\mathrm{d}}$. The parameter vector m contains the slip of each spatiotemporal unit.

Assuming Gaussian distributed data noise, the likelihood function $P(\mathbf{d} \mid \mathbf{m})$ in eq. (1) can be written as

$$
\begin{aligned}
P(\mathbf{d} \mid \mathbf{m})= & (2 \pi)^{-N / 2}\left|\mathbf{C}_{\mathbf{d}}\right|^{-1 / 2} \\
& \exp \left[-\frac{1}{2}(\mathbf{G m}-\mathbf{d})^{T} \mathbf{C}_{\mathbf{d}}{ }^{-1}(\mathbf{G m}-\mathbf{d})\right],
\end{aligned}
$$

where $\mathbf{C}_{\mathbf{d}}$ is the data covariance matrix, $|\cdot|$ denotes the determinant, $(\cdot)^{T}$ the matrix transpose and the data vector $\mathbf{d}$ includes the concatenated seismograms. Matrix $\mathbf{G}$ is of size $N \times M$ and contains Green's functions for each parameter. The data covariance matrix contains information about both measurement and modelling errors (e.g. Dettmer et al. 2007) and its detailed estimation can be quite complex. In this work we will assume only simple forms of the data covariances. For more advanced treatments, the reader is referred to Yagi \& Fukahata (2011), Duputel et al. (2012, 2014), Hallo \& Gallovič (2016) and Dettmer et al. (2012, 2014).

The prior $P(\mathbf{m})$ quantifies information about the parameters that is independent of the data information (e.g. physical or geological constraints). Thus, it is natural to incorporate positivity constraints directly into the prior. In principle, this can be achieved by utilizing any multivariate PDF with positive support. In practice, a truncated multivariate Gaussian has been normally employed to this end (e.g. Fukuda \& Johnson 2008; Kubo et al. 2016; Nocquet 2018), probably since the optimization problem that results from finding the MAP can be addressed with the familiar NNLS. The truncated Gaussian prior can be written, based on an untrucated Gaussian of zero mean, as

$P(\mathbf{m})=\left\{\begin{array}{c}(\pi / 2)^{-M / 2}\left|\mathbf{C}_{p}\right|^{-1 / 2} \exp \left[-\frac{1}{2} \mathbf{m}^{T} \mathbf{C}_{p}^{-1} \mathbf{m}\right], \\ \text { for } m_{i} \geq 0, \quad i=1, \ldots, M \\ 0 \quad \text { otherwise. }\end{array}\right.$

The matrix $\mathbf{C}_{\mathrm{p}}$ corresponds to the covariance matrix of the untruncated Gaussian PDF that results from dropping the positivity constraints in eq. (3) and contains the prior information about parameter correlations. A common approach is to enforce spatial slip correlations through a linear Laplacian smoothing operator (e.g. Yabuki \& Matsu'ura 1992; Tarantola 2005; Fukuda \& Johnson 2008; Kubo et al. 2016) say $\mathbf{L}$, of size $M \times M$ and set

$\mathbf{C}_{\mathrm{p}}^{-1}=\frac{\mathbf{L}^{T} \mathbf{L}}{\alpha^{2}}$,

where $\alpha$ is an adjustable parameter that controls how informative the prior is. Other prior correlation conditions can also be implemented by replacing $\mathbf{L}$ with alternative operators. For instance, $\mathbf{L}$ $=\mathbf{I}$ results in a minimum norm prior with no correlations, equivalent to minimum model regularization. Importantly, $\mathbf{C}_{p}$ must be a 
positive definite matrix so that $P(\mathbf{m} \mid \alpha)$ is a proper PDF that ensures that $\mathbf{C}_{p}$ possesses a Cholesky decomposition. Thus, for an arbitrary $\mathbf{C}_{\mathrm{p}}$ one can choose $\mathbf{L}$ as the Cholesky factorization of its inverse. This property can be used, for instance, to realize more general covariances such as Von Karman regularization, recently employed by Amey et al. (2018).

The posterior PDF can then be computed from eqs (2) and (3) using Bayes' theorem (eq. 1) as

$P(\mathbf{m} \mid \mathbf{d}, \alpha)=\left\{\begin{array}{c}C_{1} \exp \left(-\frac{\psi(\mathbf{m})}{2}\right), \\ \text { for } m_{i} \geq 0, \quad i=1, \ldots, M \\ 0 \quad \text { otherwise, }\end{array}\right.$

where $C_{1}$ is the normalization constant given by

$C_{1}=\left[\int_{\mathbf{m} \geq \mathbf{0}} d \mathbf{m} \exp \left(-\frac{\psi(\mathbf{m})}{2}\right)\right]^{-1}$,

and $\psi(\mathbf{m})$ is the objective function that includes the Gaussian prior

$\psi(\mathbf{m})=(\mathbf{G m}-\mathbf{d})^{T} \mathbf{C}_{\mathbf{d}}{ }^{-1}(\mathbf{G m}-\mathbf{d})+\alpha^{-2} \mathbf{m}^{T} \mathbf{C}_{p}^{-1} \mathbf{m}$.

The integral in eq. (6) is known as the marginal likelihood or evidence. Traditional inversion approaches estimate an optimal model, subject to positivity constraints, that minimizes $\psi$ for an optimal value of $\alpha$. Such a value is typically found by utilizing a grid search. Since the optimal model maximizes the posterior PDF given by eq. (5), it corresponds to the MAP solution of a Bayesian approach. However, unless the full posterior PDF is examined, other inferences cannot be obtained. Examples of quantities that require the full posterior PDF are: the mean solution, parameter correlations and parameter uncertainties.

A remaining issue is the dependence on the hyperparameter $\alpha$. Most commonly, optimal values are determined by grid search and the application of some criterion that addresses the intrinsic tradeoff between fitting the data and model smoothness. These criteria include the L-curve test (e.g. Hansen 1992), the discrepancy principle (Constable et al. 1987; Aster et al. 2005) and generalized cross-validation (e.g. Craven \& Wahba 1978). From the Bayesian point of view, $\alpha$ can be treated as an unknown nuisance variable to be estimated from the data since Bayes' theorem intrinsically and quantitatively addresses the smoothness and data fit trade-off (MacKay 2003). The ABIC method achieves this by estimating an optimal $\alpha$ value based on an analytic expression for the evidence.

Fukuda \& Johnson (2008) note that the ABIC should not be applied in FFI with positivity constraints, and that it does not give optimal results for smoothing. The main issue is that the ABIC requires computation of the marginal likelihood (see eq. 6), which is not available analytically when positivity constraints are applied. Similarly, the computation of the posterior covariance matrix

$\mathbf{C}_{\mathbf{m}}=\int d \mathbf{m}(\mathbf{m}-\hat{\mathbf{m}})^{T}(\mathbf{m}-\hat{\mathbf{m}}) P(\mathbf{m} \mid \mathbf{d})$,

where $\hat{\mathbf{m}}$ is the posterior mean, cannot be obtained analytically because $P(\mathbf{m} \mid \mathbf{d})$ is not analytic and model uncertainties require numerical estimation due to the choice of a truncated Gaussian prior. To overcome this difficulty, Fukuda \& Johnson (2008) implement an MCMC sampling method to estimate the posterior PDF by MCMC sampling. This sampling has orders of magnitude higher computational cost since $\psi$ needs to be computed for large numbers of parameter vectors. Recently, Nocquet (2018) has proposed a more efficient approach to work with the posterior given by eq. (5) that does not require an MCMC sampling. In that work, the parameter marginals and covariances are approximated by exploiting semianalytical results for the truncated Gaussian distribution (Genz \&
Bretz 2009) and the MAP is obtained from the NNLS. As we discuss below, instead of approximating directly the posterior in eq. (5), we opt for performing a parameter transformation to logarithmic space and approximating the resulting posterior PDF with a multivariate Gaussian.

\subsection{Positivity by variable transformation}

The approach described in Section 2.1 provides robust estimates of the posterior PDF but the only way to compute it is by the MCMC sampling. However, under some circumstances the computational infrastructure for the MCMC sampling may not be available and some applications, such as disaster response, require rapid finite fault solutions. In these situations, it is also important to understand parameter uncertainties that can reduce the risk of basing decisions on misleading solutions (e.g. misplacing shallow slip or under/overestimating peak slip). Therefore, it is desirable to apply Bayesian methods that provide uncertainties efficiently based on approximations.

To avoid the MCMC sampling, we apply two changes in the approach previously described (Section 2.1): First, positivity is implemented by a variable transformation that considers the natural logarithm of each parameter instead of the parameters themselves. Second, the posterior in logarithmic space is approximated as a Gaussian around the MAP solution. The first change is required to provide positivity for which an analytic estimate of the posterior PDF exists. This is particularly important for subfaults with near-zero slip, where a Gaussian PDF approximation would lead to erroneous results. In contrast, by working in logarithmic space, the new parameters are defined for all real numbers and truncation is not a problem. This idea has been previously proposed by Tarantola (2005; section 3.2.1) and we preliminary apply it to W-phase FFI (Benavente 2016, Benavente et al 2015 ). To our knowledge, it has not been applied elsewhere to FFI.

To formally state our algorithm, we define the parameter transformation from slip to logarithmic slip as

$\mathbf{s}(\mathbf{m})=\left[\ln m_{1}, \ln m_{2}, \ldots, \ln m_{M}\right]^{T}$

and

$\mathbf{e}(\mathbf{s})=\left[\exp s_{1}, \exp s_{2}, \ldots, \exp s_{M}\right]^{T}$.

Solving for $\mathbf{s}$ intrinsically leads to non-negative parameter values in $\mathbf{m}$ and we formulate the inversion in terms of parameters $\mathbf{s}$. Importantly, both $\mathbf{e}(\mathbf{s})$ and $\mathbf{m}$ represent the same parameter vector, however m is not intrinsically positive so positivity must be explicitly enforced as a separated condition when working in terms of $\mathbf{m}$ (see eq. 3). The likelihood function is given by

$$
\begin{aligned}
P(\mathbf{d} \mid \mathbf{s})= & (2 \pi)^{-N / 2}\left|\mathbf{C}_{\mathbf{d}}\right|^{-1 / 2} \\
& \exp \left[-\frac{1}{2}[\mathbf{G e}(\mathbf{s})-\mathbf{d}]^{T} \mathbf{C}_{\mathbf{d}}{ }^{-1}[\mathbf{G e}(\mathbf{s})-\mathbf{d}]\right] .
\end{aligned}
$$

In contrast to the truncated Gaussian prior discussed below (eq. 3), we employ a multivariate lognormal prior for the slip parameters $\mathbf{m}$ that naturally incorporates positivity constraints. Consequently, the prior for the logarithmic slip parameters $\mathbf{s}$ is given by a multivariate normal distribution:

$P(\mathbf{s} \mid \tilde{\alpha})=\left(2 \pi \tilde{\alpha}^{2}\right)^{-M / 2}\left|\mathbf{L}^{T} \mathbf{L}\right|^{1 / 2} \exp \left[-\frac{1}{2 \tilde{\alpha}^{2}}(\mathbf{L s})^{T}(\mathbf{L s})\right]$,

whose covariance matrix is given by

$\tilde{\mathbf{C}}_{p}^{-1}=\frac{\mathbf{L}^{T} \mathbf{L}}{\tilde{\alpha}^{2}}$. 
In our application, $\mathbf{L}$ is the linear Laplacian smoothing operator and, thus, $\tilde{\alpha}$ correspond to the smoothing parameter in logarithmic space. Note that this smoothing prior is applied in logarithmic space and not equivalent to that in eqs (3) and (4). This implies that slip models obtained from the two approaches may exhibit different spatial slip correlations. In particular, since the stress drop on the fault can be related to such correlations, both approaches may yield dissimilar stress drops for the same event. We note, however, that a priori knowledge about the stress drop can be difficult to establish. In this sense, smoothing in logarithmic space appears to be a reasonable choice since it may allow abrupt spatial changes in slip, if data support them, and in Section 3 we carry out a comparison of the two smoothing approaches.

Using the Bayes' theorem (eq. 1) and eqs (11) and (12), we obtain

$P(\mathbf{s} \mid \mathbf{d}, \tilde{\alpha})=C_{2} \exp \left(-\frac{\tilde{\psi}(\mathbf{s})}{2}\right)$

where

$C_{2}=\left[\int d \mathbf{s} \exp \left(-\frac{\tilde{\psi}(\mathbf{s})}{2}\right)\right]^{-1}$

and

$\tilde{\psi}(\mathbf{s})=(\mathbf{G e}(\mathbf{s})-\mathbf{d})^{T} \mathbf{C}_{\mathbf{d}}{ }^{-1}(\mathbf{G e}(\mathbf{s})-\mathbf{d})+\tilde{\alpha}^{-2}(\mathbf{L s})^{T}(\mathbf{L s})$.

The integral in eq. (15) is taken over all real numbers since $\mathbf{s}$ is an unbounded quantity, in contrast to $\mathbf{m}$. Note that the new objective function is now nonlinear and requires iterative linearization for efficient solving. The main assumption in our method is that the posterior in eq. (14) can be approximated by a Gaussian as discussed below.

\subsection{Uncertainty estimation by linearization}

Slip uncertainties can be inferred from the model covariance matrix defined by eq. (8). In principle, this requires numerical integration to evaluate eq. (8) using eq. (14). However, we can linearize the problem by approximating the posterior by a Gaussian PDF locally around the MAP solution of $\mathbf{s}$, a procedure that is commonly applied to weakly nonlinear inverse problems (e.g. Sivia \& Skilling 2006). In Section 3, we evaluate the accuracy of this approximation and in the following we discuss its application to our problem.

For a PDF similar to eq. (14) to be reasonably approximated by a Gaussian, a sufficient condition is that the objective function $\tilde{\psi}$ is a quadratic function of $\mathbf{s}$ (e.g. Tarantola 2005, section 6.21). For linear forward problems this condition is satisfied exactly. When the forward problem is nonlinear, the strategy is to expand the objective function around the MAP and retain terms up to second order. For highly nonlinear problems, higher-order terms may be significant and a second-order approximation may be poor. A second-order approximation is given by

$\tilde{\psi}(\mathbf{s})=\tilde{\psi}(\hat{\mathbf{s}})+\frac{1}{2}(\mathbf{s}-\hat{\mathbf{s}})^{T} \nabla \nabla \tilde{\psi}(\hat{\mathbf{s}})(\mathbf{s}-\hat{\mathbf{s}})$,

where $\nabla \nabla \tilde{\psi}$ is the Hessian matrix of $\tilde{\psi}$ and $\hat{\mathbf{s}}$ is the MAP solution. The first-order term is missing from eq. (17) because it includes the factor $\nabla \tilde{\psi}(\hat{\mathbf{s}})$, which is the gradient of $\tilde{\psi}$ at the MAP, which must vanish at a minimum in $\psi(\mathbf{s})$.

Substituting eq. (17) in eq. (14) gives

$P(\mathbf{s} \mid \mathbf{d}, \tilde{\alpha}) \propto \exp \left[-\frac{1}{2}(\mathbf{s}-\hat{\mathbf{s}})^{T} \mathbf{C}_{s}^{-1}(\mathbf{s}-\hat{\mathbf{s}})\right]$, where

$\mathbf{C}_{s}^{-1}=\frac{1}{2} \nabla \nabla \tilde{\psi}(\hat{\mathbf{s}})$

is the covariance matrix of the Gaussian posterior PDF and omission of the normalizing constant causes the proportionality in eq. (18). These expressions can be evaluated analytically by computing the Hessian matrix. From eq. (16), the gradient and Hessian of the objective function are

$\nabla \psi(\mathbf{s})=2 \mathbf{e} \otimes \mathbf{G}^{T} \mathbf{C}_{\mathbf{d}}^{-1}(\mathbf{G e}-\mathbf{d})+2 \alpha^{-2} \mathbf{L}^{T} \mathbf{L s}$

and

$$
\begin{aligned}
\nabla \nabla \psi(\mathbf{s})= & 2 \mathbf{e e}^{T} \otimes \mathbf{G}^{T} \mathbf{C}_{\mathbf{d}}{ }^{-1} \mathbf{G} \\
& +2 \operatorname{diag}\left[\mathbf{e} \otimes \mathbf{G}^{T} \mathbf{C}_{\mathbf{d}}{ }^{-1}(\mathbf{G e}-\mathbf{d})\right] \\
& +2 \alpha^{-2} \mathbf{L}^{T} \mathbf{L}
\end{aligned}
$$

respectively. For convenience we do not write explicitly the dependence $\mathbf{e}(\mathbf{s})$. The symbol $\otimes$ denotes the element-wise product and the operator diag creates a diagonal matrix whose elements are the elements of the vector argument. Derivations for eqs (20) and (21) are given in the Appendix A.

\subsection{Inversion strategy}

The goal of our work is to obtain an analytic approximation of the posterior PDF so that uncertainties can be estimated. The procedure can be summarized as follows: First, we parametrize the rupture process following the MTWM. This involves prescribing the number and geometry of subfaults, the number of time windows and the maximum rupture velocity. In this study, we assume that a data covariance matrix $C_{\mathrm{d}}$ and an optimal value for $\alpha$ have been previously estimated and are known. Since directly determining these parameters as part of the inversion results in a nonlinear inverse problem, iterative approaches to estimate them are used instead (e.g. Benavente et al. 2016).

After parametrizing the problem, we proceed to find the MAP solution. The optimization is carried out by minimizing the objective function $\tilde{\psi}$. Because of its nonlinearity (see eq. 16), an iterative approach is applied. Since analytic expressions exist for the linearized gradient and Hessian (eqs 20 and 21, respectively), efficient methods can be applied to find the minimum (e.g. Press et al. 1992; Aster et al. 2005). In this work, we use the Newton conjugate gradient method (Newton-CG; Wright \& Nocedal 1999) as implemented by SciPy (Jones et al. 2001).

As for any other nonlinear inversion, some concern remains regarding the uniqueness of the solution. If $\tilde{\psi}$ is a convex function the solution is guaranteed to be unique. While we do not provide a general proof of convexity, the objective function appears empirically well behaved near the MAP solution. We reach this conclusion since a sufficient requirement for convexity is a positive definite Hessian. Eq. (21) shows that the Hessian comprises three terms. The first and third terms are positive definite as long as the rows of $\mathbf{G}$ and $\mathbf{L}$ are linearly independent. However, the second term can contribute negative elements to the diagonal which may cause convexity issues. Nevertheless, for solutions close to the MAP the predictions should be comparable to the data and since the contribution of the second term to the Hessian is modulated by the residual vector $(\mathbf{G e}-\mathbf{d})$, it should be small compared to the other two. Furthermore, all the Hessian matrices we obtained for MAP solutions in this work were positive definite.

After obtaining the MAP, we calculate the posterior covariance matrix from eq. (19) employing the Hessian from eq. (21), and 
then we use this covariance matrix to estimate the uncertainties. At this point, we have approximate expressions for the posterior PDF in logarithmic space given by eq. (18). In principle, the diagonal elements of the covariance matrix are sufficient to obtain marginal distributions for single parameters since the marginals of a Gaussian are also Gaussian (e.g. Tarantola 2005; section 6.5).

However, to interpret the solution in terms of slip in linear space, a coordinate transformation to $\mathbf{e}$ is required. To consider the transformation of uncertainties, we note that normally distributed random variables in logarithmic space have log-normal distribution in linear space. That is to say if $x=\ln y$ is normally distributed with mean $\mu$ and standard deviation $\sigma, y$ has log-normal distribution as

$P(y)=\frac{1}{y \sigma \sqrt{2 \pi}} \exp \left[-\frac{(\ln y-\mu)^{2}}{2 \sigma^{2}}\right]$.

Note the convention that $\mu$ and $\sigma$ are the parameters of the normal distribution in logarithmic space. The mean $\Omega$ and the standard deviation $\Sigma$ of $P(y)$ are given by

$\Omega=e^{\mu+\sigma^{2} / 2}$

and

$\Sigma=\sqrt{\left(e^{\left.\sigma^{2}+2 \mu\right)}\left(e^{\sigma^{2}}-1\right)\right.}$.

As a measure of uncertainties in the log-normally distributed parameters we use the width of the 95 percent credibility interval (CI). Since the log-normal distribution is asymmetric (in contrast to the normal distribution), the use of CIs to quantify uncertainties is preferable to the use of the standard deviations. The CIs are computed directly by sampling the approximated posterior as discussed in Section 3.2.

\section{INVERSION FOR SIMULATED DATA}

This section applies our method to simulated noisy data to study whether it can provide meaningful slip uncertainties. Also, we study the sensitivity of the observations to the model parameters by reducing the station azimuthal coverage. To this end, we employ synthetic surface waves based on the target model in Fig. 1. Long period Love and Rayleigh waves are computed by using the method of Kanamori \& Stewart (1976), accounting for phase velocity changes that are caused by 3-D structure (Ekström et al. 1997). Synthetic waveforms are bandpass filtered from 100 to $200 \mathrm{~s}$. We first assess the effects of our smoothing scheme in logarithmic space. Then, we consider uncertainties for simulated data with different noise levels. Finally, we compare the linearized uncertainty estimates from our method to those obtained by the MCMC sampling.

\subsection{Effects of smoothing in logarithmic space}

An important consequence of the variable transform we apply is that smoothing is applied in logarithmic space. Typical FFIs apply smoothing directly to slip or scalar moment. While it is reasonable to assume some form of smoothness for slip, the precise implementation is subjective. In this simulation, we study to which extent log-space smoothing is different from non-logarithmic smoothing which is widely applied. Here, we establish that logarithmic smoothing can recover the main features of a slip model that satisfies the traditional linear smoothing assumption.

The simulation geometry is based on the 2015 Illapel event (Chile, $M_{\mathrm{w}}=8.3$ ) and consists of 16 subfaults along strike $\left(4^{\circ}\right)$ and 6 along $\operatorname{dip}\left(18^{\circ}\right)$. Only one time window of $10 \mathrm{~s}$ half duration was employed resulting in constant rupture velocity. The model includes only positive slip values that are decomposed into two rake components $\left(45^{\circ}\right.$ and $\left.135^{\circ}\right)$. This is a very simple parametrization intended only to produce a workable example for simulation purposes. A more realistic parametrization was prepared for our application with observed data and it is presented in Section 4. Importantly, the model satisfies a traditional smoothing scheme (not in log-space) expressed by the prior of eq. (3). Fig. 1 shows the target slip distribution. The model is clearly smooth and exhibits a major slip patch at the centre of the fault and much smaller variations in slip outside this main slip patch.

We compute synthetic surface wave data based on the target model and stations shown in Fig. 1. Then, we add correlated Gaussian noise and invert the resulting simulated data using our algorithm. To achieve a temporal correlation that is consistent with the frequency content in the synthetics, an exponential correlation matrix $\mathbf{C}_{\text {syn }}$ is introduced in the form of a block diagonal matrix. Each block $\mathbf{C}_{\mathrm{syn}}^{i}$ contains correlation information for a given station $i$ and its components are

$\left[\mathbf{C}_{\mathrm{syn}}^{i}\right]_{k l}=\sigma_{i}^{2} \exp \left(\frac{-\left|t_{k}-t_{j}\right|}{\tau}\right)$,

where $t_{i}$ is the time of the $i$ th sample of the waveform. The scaling parameter $\sigma_{i}^{2}$ controls the noise level and we compute it as $\sigma_{i}^{2}=$ $\gamma A_{i}^{\max }$ with $A_{i}^{\max }$ the maximum absolute value of the amplitude for station $i$ and $\gamma$ a parameter controlling the absolute noise level of the traces. The temporal correlation decay $\tau$ is fixed at $100 \mathrm{~s}$, in agreement with the minimum period content in the signals. Once $\mathbf{C}_{\text {syn }}$ is computed, correlated noise is obtained by multiplying its Cholesky decomposition with a vector of standard uncorrelated Gaussian samples. Fig. 2 shows an example of the synthetic traces generated by this procedure for $\gamma=5$ per cent.

We consider two noise levels that are given by $\gamma=2$ per cent and $\gamma=5$ per cent. Smoothness was adjusted following the discrepancy principle (Constable et al. 1987; Aster et al. 2005) for the known covariance matrix, that is, the logarithmic smoothness is such that the $\chi^{2}$ misfit equals the number of data points, with

$\chi^{2}=[\mathbf{G e}(\mathbf{s})-\mathbf{d}]^{T} \mathbf{C}_{\mathbf{d}}{ }^{-1}[\mathbf{G e}(\mathbf{s})-\mathbf{d}]$.

The results are given in Fig. 3. While both results agree well with the true model, both are less smooth and display more abrupt changes in the slip distribution than the true model. Also, peak slip is larger $(\sim 3.2 \mathrm{~m}$ ) for the noisiest model while the slip region is nearly the same for the three models. However, these differences are small compared to the slip uncertainties of the solution considered in Section 3.2. We conclude that log-space smoothing achieves results that are similar to smoothing in linear space.

\subsection{Uncertainty estimation for different noise levels}

In a Bayesian framework, results are given in terms of a posterior PDF that includes information about the possible models that can produce the data. For our application, the estimation of slip uncertainties is straightforward once an MAP solution is obtained. Eq. (19) is evaluated at the MAP solution to approximate the posterior by a Gaussian PDF in the s variables. The single-parameter marginals of this multivariate Gaussian are also Gaussian distributions with variance given by the appropriate diagonal elements of the covariance matrix. Then, the marginals of each parameter in linear space are given by log-norm distributions in e with mean and standard deviation given by eqs (23) and (24), respectively. While all the relevant statistics (e.g. CIs and mean) can be derived for 

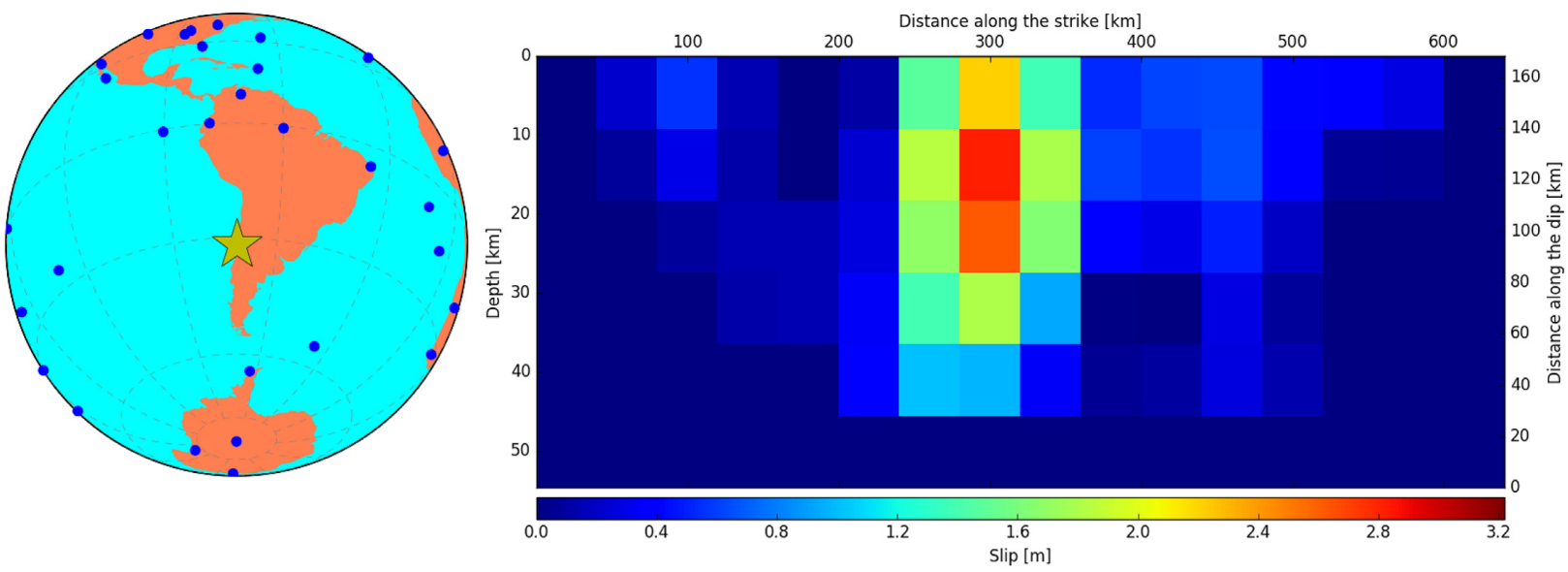

Figure 1. Target model for the simulations. Left-hand side: Stations (blue), hypocentre (star) and fault geometry based on the 2015 Illapel earthquake. The true slip distribution (right-hand side) is chosen to be representative for an event of this size while maintaining high degree of smoothness.
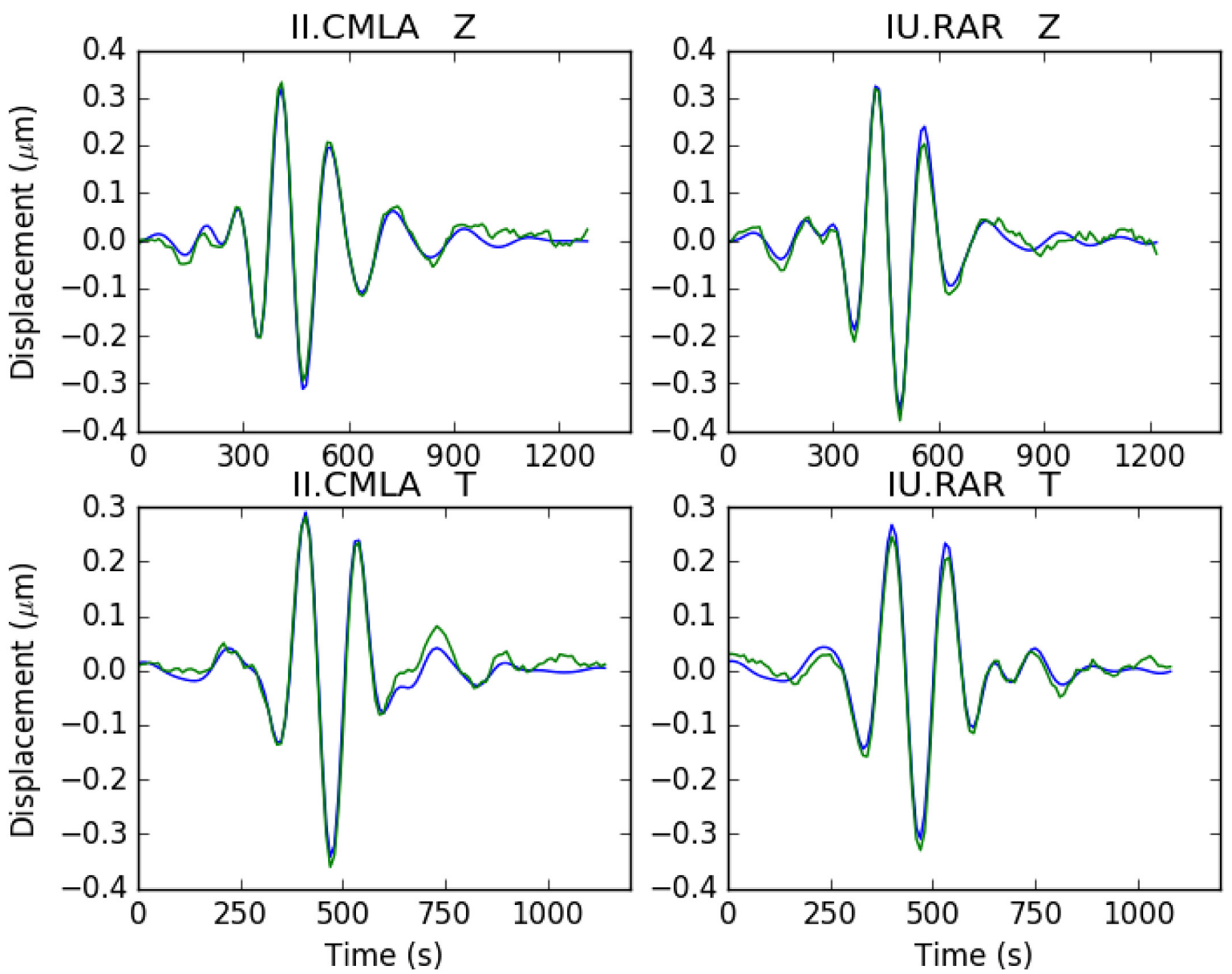

Figure 2. Example of a synthetic target (blue) and noisy (green) traces employed in the simulation for $\gamma=5$ per cent. For each subplot, station name and component (T: Transverse, Z: Up) are displayed at the top.

the parameters from these marginals, their physical interpretation is difficult because of the parametrization scheme (i.e. MTWM).

In order to obtain uncertainties for the cumulative slip distribution, an additional step is required. This is because in the MTWM, the parameters given by $\mathbf{m}$ correspond to the slip of each spatiotemporal unit that are also decomposed into rake directions. That is, the cumulative slip (or scalar moment) $S_{i}^{\text {cum }}$ of a subfault $i$ is

$$
S_{i}^{\text {cum }}=\sqrt{\left(\sum_{j}^{N_{t}} S_{i}^{j 1}\right)^{2}+\left(\sum_{j}^{N_{t}} S_{i}^{j 2}\right)^{2}},
$$



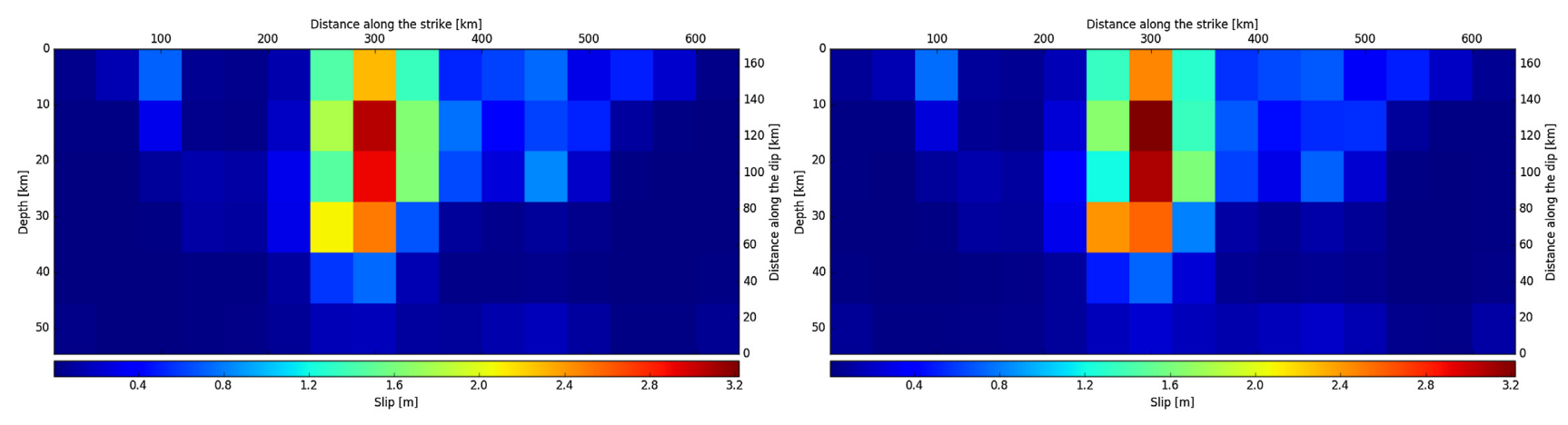

Figure 3. Simulation results for the log-space smoothing approach in terms of the MAP model. Results for $\gamma=2$ per cent (left-hand panel) and $\gamma=5$ per cent (right-hand panel) noise level are shown. Both results agree well with the true model (Fig. 1).

where $S_{i}^{j 1}$ and $S_{i}^{j 2}$ are the rake components for the $j$ th time window and $N_{\mathrm{t}}$ is the number of time windows. Thus, it is possible to propagate the uncertainties from parameters marginals to cumulative slip analytically. Here, however, we follow a rather simpler sampling approach. Since drawing samples from a multivariate Gaussian PDF is an inexpensive procedure, we draw $N_{\text {samp }}$ samples directly from $P(\mathbf{s} \mid \mathbf{d}, \tilde{\alpha})$. For each of those samples we apply eq. (27) obtaining the respective cumulative slip. We then compute the 95 per cent CIs from the cumulative slip samples. Our tests show that $N_{\text {samp }} \approx 10^{5}$ provide stable CI to an accuracy of $\sim 1 \mathrm{~cm}$, that is, results do not change above this limit as more sampling is done.

The 95 per cent CIs of the cumulative slip distribution (Fig. 3) are shown in Fig. 4. The uncertainty patterns are similar, but the peak uncertainty for noise level $\gamma=2$ percent is $\sim 1.8 \mathrm{~m}$ and for $\gamma=5$ per cent uncertainty reaches $\sim 2.7 \mathrm{~m}$. The results suggest that surface waves tend to lose resolution at larger depths but constrain shallow slip well. However, slip for the bottommost subfaults appears to be relatively well constrained and close to $0 \mathrm{~m}$, suggesting that the data are sensitive to the total rupture width. While these uncertainty estimates appear reasonable, we carry out a quantitative comparison with the MCMC sampling as an independent method in Section 3.3. It should be noticed that slip uncertainties will not only depend on the data noise level but also on the station distribution and azimuthal coverage around the source. As an example, we repeat the simulation for $\gamma=5$ per cent but filtering out all stations in the eastern hemisphere with respect to the epicentre. Uncertainties are shown in Fig. 5 and the mean model in Fig. 6. As expected, uncertainties are significantly larger, with a peak 95 percent CI of $3.6 \mathrm{~m}$. Again, uncertainties are larger downdip but data constrain the shallowest portion of the fault well.

\subsection{Comparison with the MCMC sampling}

This section applies the MCMC sampling to obtain nonlinear uncertainty estimates and compares these to the linearized results of the previous section. The comparison is based on the simulated surface wave data with $\gamma=5$ per cent noise level. Noise correlations are not introduced so that synthetic traces were generated from a covariance given by $\mathbf{C}_{\text {syn }}^{i}=\sigma_{i}^{2} \mathbf{I}^{\left(n_{i}\right)}$, where $n_{i}$ is the number of data points of the $i$ th trace. The uncertainty estimates are considered separately for each rake component. The resulting log-normal marginal distributions are shown as the red curves in Fig. 7 for rake component 1 $\left(45^{\circ}\right)$ and Fig. 8 for rake component $2\left(135^{\circ}\right)$.

An MCMC sampling algorithm is applied to obtain independent marginal distributions for the parameters. In this approach, random samples are drawn directly from the nonlinear posterior PDF (eq. 14). Here, we employ a fixed-dimension version of the transdimensional sampling algorithm by Dettmer et al. (2014). To isolate the effect of the linearization approximation, we fix the smoothing parameter $\alpha$ to the same value we estimated in our linearized method. The MCMC sampling results in a large ensemble of parameter vectors that numerically approximate the posterior. Marginal distributions are straightforward to obtain as normalized histograms for each parameter. These marginal PDFs are shown for the appropriate rake component in Figs 7 and 8 (blue histograms).

In general, we find very good agreement between the marginals obtained by the two methods (Figs 7 and 8). The histograms reveal that most parameters do not exhibit features indicating strong nonlinearity (such as multiple modes or other complicated shapes). Rather, the log-normal approximation seems appropriate with similar peak positions and uncertainties throughout. However, we note that fixing $\alpha$ to an optimal value can impact both estimated slip values and uncertainties. In practice, treating $\alpha$ as unknown in the MCMC sampling provides a more general solution than possible in a linearized approach. We conclude that the comparison indicates that our approximation can recover meaningful uncertainty estimates at a fraction of the computational expense required by nonlinear methods. For this particular example the MCMC sampling method converged to the posterior shown after $12 \mathrm{hr}$ using 12 CPUs while our linearized approach only requires seconds on a desktop computer.

\section{APPLICATION: ILLAPEL GREAT EARTHQUAKE (2015, $\left.M_{\mathrm{W}}=8.3\right)$, CHILE}

In this section, we apply our inversion approach to estimate the slip distribution of the 2015 Illapel earthquake $\left(M_{\mathrm{w}}=8.3\right)$ and its uncertainty. Early finite fault models inferred peak slip of 5-10 m (Ye et al. 2015; Benavente et al. 2016; Melgar et al. 2016; Tilmann et al. 2016) and the maximum tsunami run-up was estimated to be $10.8 \mathrm{~m}$ (Aránguiz et al. 2016). As discussed in previous sections, to utilize our inversion approach we parametrize the rupture process with the MTWM. For the Illapel event, we prescribe a maximum rupture velocity to be of $2.0 \mathrm{~km} \mathrm{~s}^{-1}$ and the source time function is discretized into three isosceles triangles with an overlap and half duration equal to $10 \mathrm{~s}$, with a maximum rupture time of $40 \mathrm{~s}$ per subfault. We employ surface waves from the station distribution shown in Fig. 1 and Green functions are generated with the approach described in Section 3. Since we correct for phase velocity changes, manual phase shifting is not introduced. The fault geometry is the same as in Section 3.1 but the number of subfaults along strike and dip is doubled for increased resolution, so that we consider 

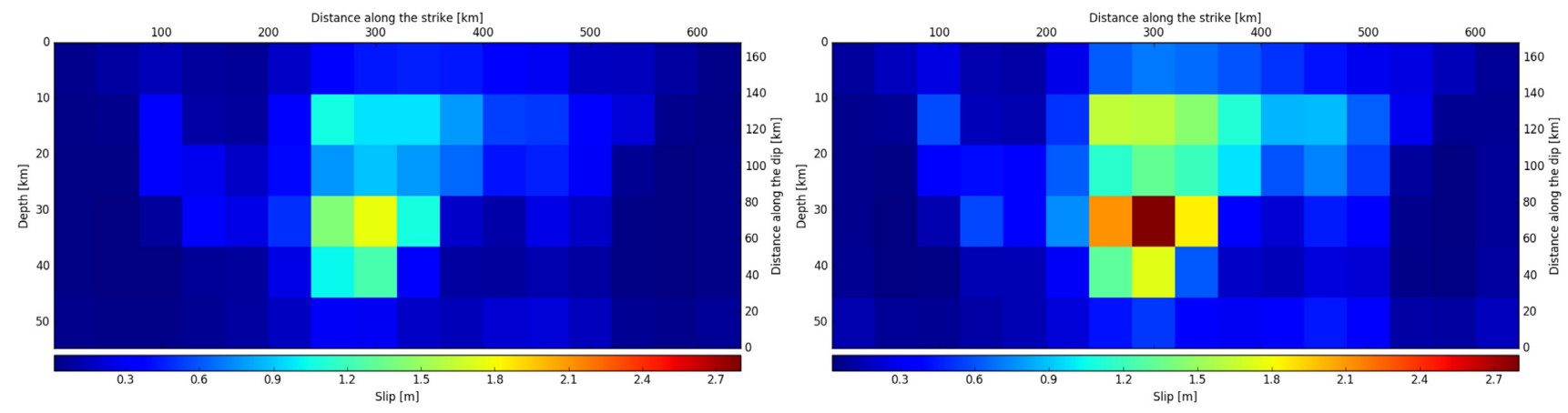

Figure 4. 95 percent CIs of recovered models using log-space smoothing. Target model is shown in Fig. 1. CIs correspond to the models of Fig. 3 in the same order, that is, $\gamma=2$ per cent of noise contamination was applied to target model for the figure on the left-hand side and $\gamma=5$ per cent for the one on the right-hand side. Results for the 2.5 and 97.5 percentiles of the PDF are shown in Figs S1 ( $\gamma=2$ per cent) and S2 of the Supporting Information $(\gamma=5$ per cent).
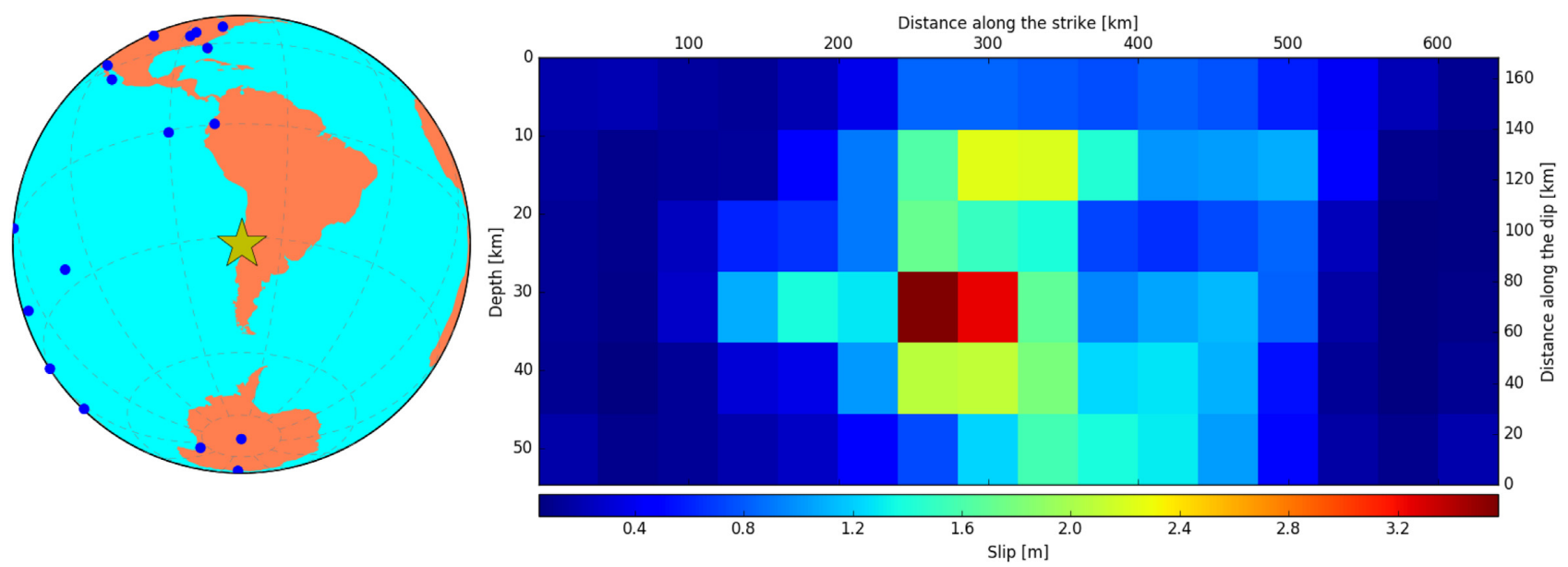

Figure 5. Station distribution and 95 per cent CIs of recovered model when stations with azimuths $0^{\circ}-180^{\circ}$ are removed from the inversion. Noise contamination is $\gamma=5$ per cent. Corresponding models for percentiles 2.5 and 97.5 are shown in Fig. S3 of the Supporting Information.

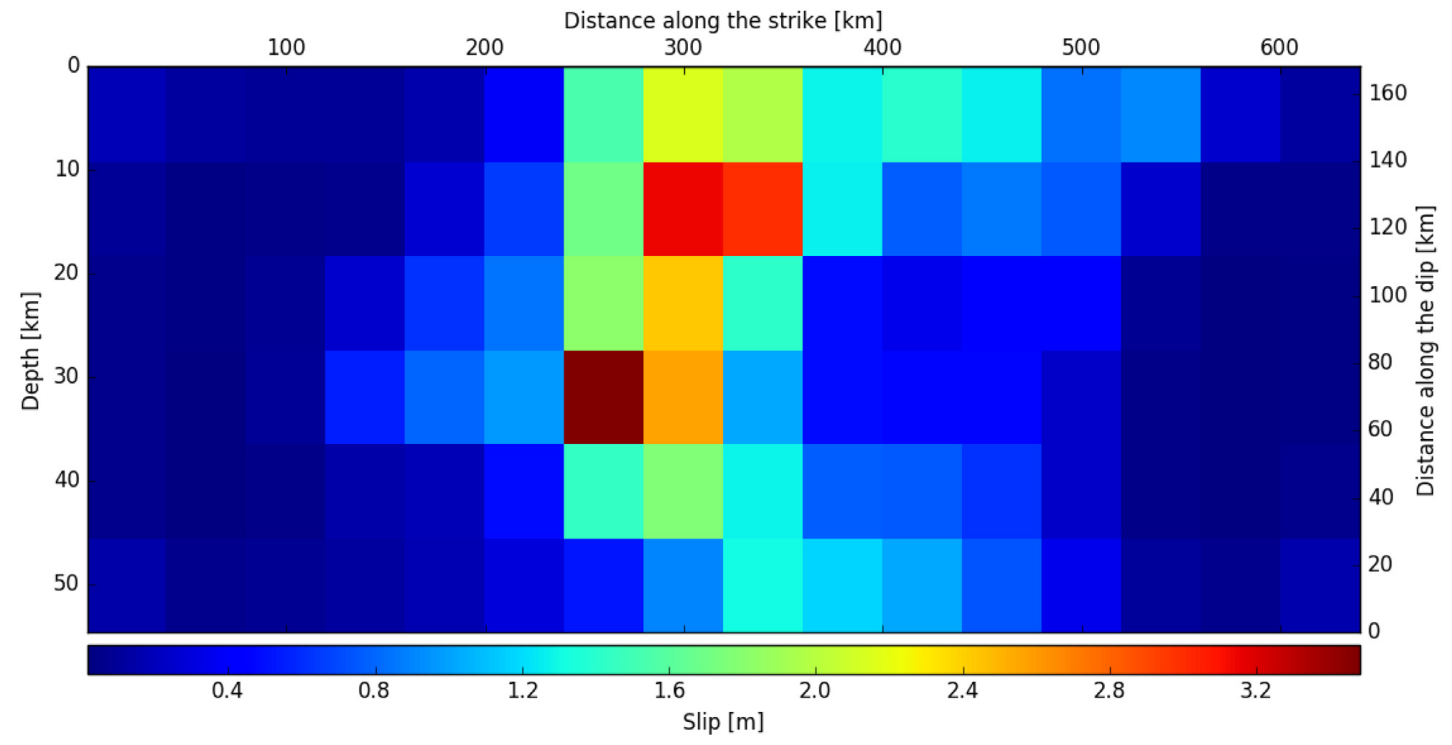

Figure 6. Mean of the cumulative slip when stations with azimuths $0^{\circ}-180^{\circ}$ are removed from the inversion. Noise contamination is $\gamma=5$ per cent.

32 subfaults along strike and 12 along dip. Rake components are taken as $45^{\circ}$ and $135^{\circ}$ so that rake angles can vary within that range. Considering rake variations within a $90^{\circ}$ range around a reference rake value $\left(90^{\circ}\right.$, in this case, as expected for a thrust event) is a common practice in FFI as it naturally results from taking two orthogonal rakes components in applying the NNLS. While sometimes rake angles are allowed to vary over a wider range of $180^{\circ}$ (e.g. Minson et al. 2013), this is not currently possible in 
Rake component 1

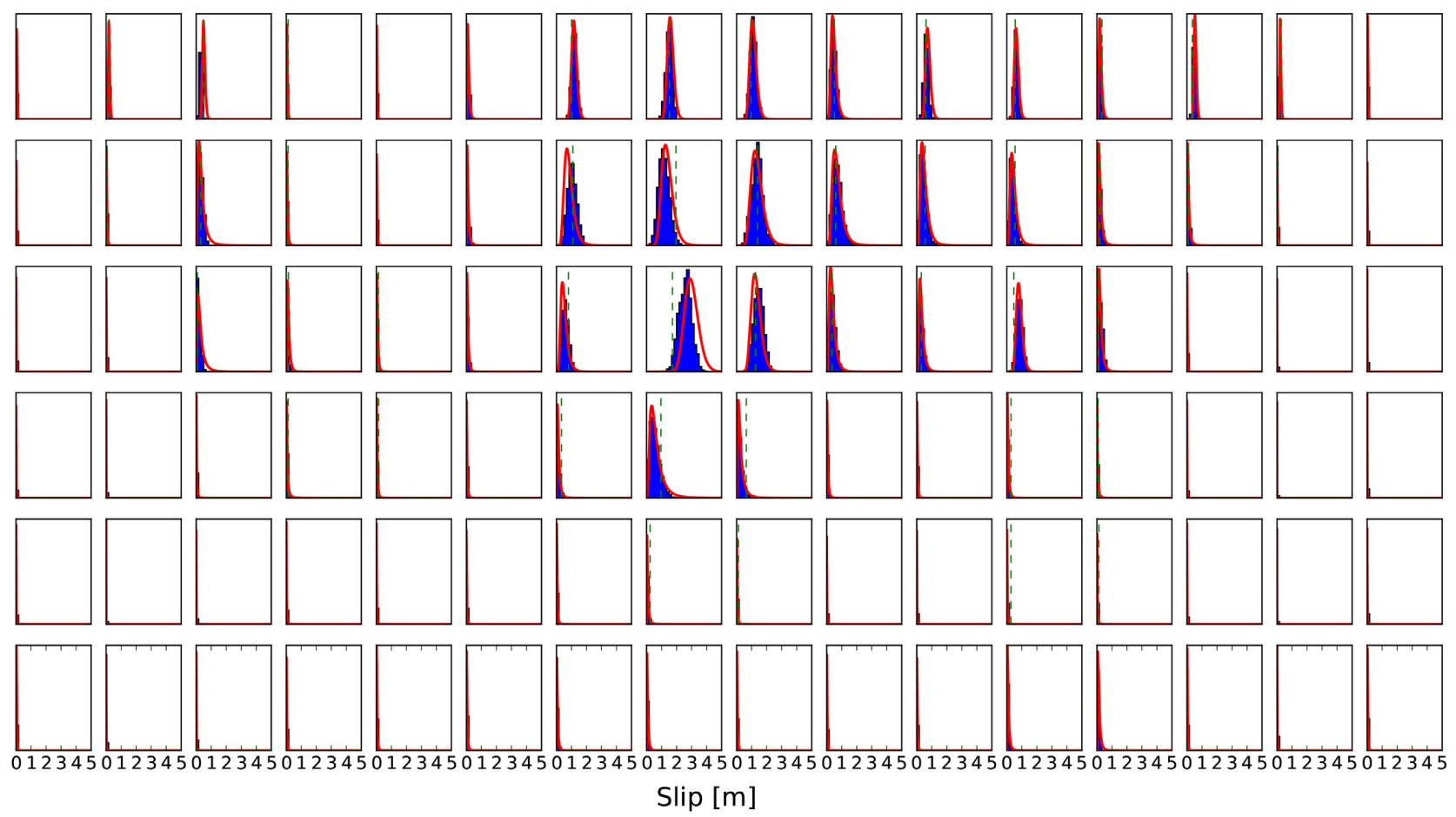

Figure 7. Marginal PDFs for parameters of rake component of $45^{\circ}$. Each subplot shows the marginal PDF for the corresponding subfaults. The layout of the subplots is the same as the slip distributions shown in Figs 1 and 4 so horizontal direction goes along strike and vertical direction along dip. The red curves were obtained using the analytic expression we proposed here and the blue histograms were obtained using the MCMC sampling of the posterior PDF. The vertical green dashed line indicate the slip of the target model (Fig. 1).

Rake component 2
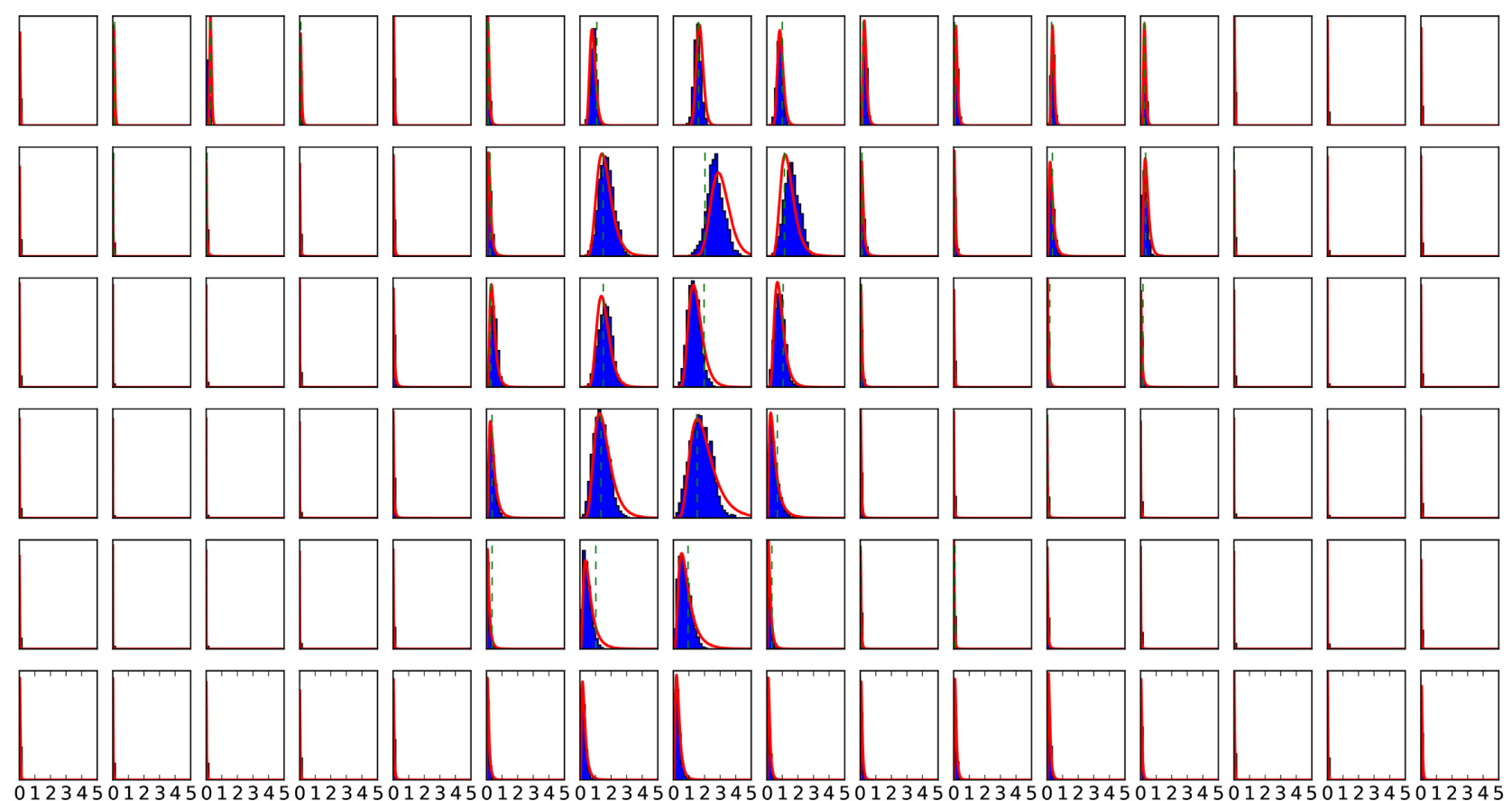

Slip $[\mathrm{m}]$

Figure 8. Marginal PDFs for parameters of rake component of $135^{\circ}$. Legends are the same as in Fig. 7 . 
our algorithm since it would involve mixing unbounded and positive parameters in the posterior, making the Gaussian approximation in log-space non-trivial.

Fig. 9 (left-hand panel) shows the posterior mean model for the cumulative slip distribution and rake angles following the sampling approach presented in Section 3.2. We note that to compute this posterior we draw $10^{5}$ samples of the parameter vector, which only requires $\sim 15 \mathrm{~s}$ in a standard desktop computer. In order to independently test the reliability of our solution, we conducted a probabilistic inversion for the posterior by the MCMC sampling following the same procedure described in Section 3.3. The data fits are shown in Figs S4-S6 and parameter marginals in Figs S7-S12 of the Supporting Information. As in the simulations, marginals for all parameters are in excellent agreement with our approximated posterior. For comparison, we have included two slip models derived from teleseismic data (Hayes 2015; Benavente et al. 2016). All slip models exhibit a similar peak slip located $\sim 100 \mathrm{~km}$ north of the epicentre in the shallow portion of the fault with nearly up-dip rake. This is consistent with the moderate tsunami triggered by the event. Our model displays an additional deeper slip patch with small slip and significant uncertainty. In addition, our solution is more compact, likely owing to the logarithmic smoothing. On the one hand, we note that the level of spatial detail in our slip model appears to be greater than that in Benavente et al. (2016)'s W-phase model, and less than in Hayes (2015)' combined surface and body wave model. This might reflect the relative period ranges in the data sets: the W-phase solution uses very long (200-1000 s) period waves, while our data set uses waves of 100-200 s period, and Hayes (2015) data set includes body waves of much shorter period. On the other hand, our main slip zone is much more compact than either the Benavente et al. (2016) or Hayes (2015) models. Since our data fits are generally good (see Figs S4-S6 in the Supporting Information), it is likely that data are not very sensitive to such spatial correlation details. Thus, we surmise that this difference may be due to differences in regularization assumptions not data information.

We also compute slip uncertainties, expressed as the 95 per cent CI, for the cumulative slip and 2-D marginals for the rake components of a relevant fault section (enclosed by the black rectangle in Fig. 10, left-hand panel). These results are shown by Figs 10 and 11, respectively. As mentioned previously, there are two slip patches in the mean slip distribution. The main slip feature is placed up dip with a peak slip of $\sim 8.5 \mathrm{~m}$ with a correspondent 95 per cent CI of $\sim 6 \mathrm{~m}$. The secondary slip feature, located downdip, has both peak slip and 95 per cent CI of $\sim 3 \mathrm{~m}$ suggesting that it has been poorly resolved by the data. In addition, this slip feature appears well pronounced one rake component $\left(135^{\circ}\right.$, see Fig. 11$)$ that has a large variability in the same region as shown by Fig. 11, suggested by the elongated marginals along rake component 2 . Since the other rake component seems to better constrain the slip in the same region, we expect that this secondary slip patch is an artefact, possibly caused by the loss of resolution with depth implied by our synthetics tests in Section 3). In any case, it would have little effect in the tsunami generation due to the oblique slip direction.

\section{SUMMARY AND DISCUSSION}

We developed an efficient method for slip uncertainty estimation in FFI with positivity constraints. A Bayesian framework is employed that incorporates uncertainty estimation naturally as part of the inversion. Positivity is ensured by a parameter transformation to logarithmic space that formulates the inversion in terms of intrinsically positive slip. This procedure results in a nonlinear inverse problem for which optimal parameters are estimated by applying a linearized iterative approach. The posterior PDF is approximated by a normal distribution in logarithmic space. Under this linearized approximation, an analytic expression exists for the posterior covariance matrix that provides straightforward uncertainty estimates in logarithmic space. These uncertainties correspond to log-normal distributions for slip in linear space.

Simulation results and comparison with the MCMC sampling show that our new method leads to meaningful uncertainties at a fraction of the computational effort required for numerical estimation. This suggests that the linearized approximation for the posterior PDF is sufficient for robust, rapid uncertainty estimation. However, more general results would be obtained if smoothing was treated as unknown, which moderately increases computational cost since it requires additional solutions to the nonlinear inverse problem (see Benavente et al. 2016). Slip distributions obtained for the Illapel event are consistent with previously published results but provide the additional benefit of uncertainty quantification. The Illapel slip uncertainties we obtained are meaningful and do not exceed MAP values in peak-slip areas (i.e. information is inferred from the data) and correlate with slip. It should be noted, however, that uncertainties are not negligible at all. While the peak slip we obtained is around $8.5 \mathrm{~m}$, the slip range in this area, estimated from the 95 per cent CI, is $\sim 6-12 \mathrm{~m}$ (see Fig. S13, Supporting Information). Such large variability can have a tremendous impact in tsunami intensities derived from the ensemble of slip models (Cienfuegos et al. 2018). Thus, we stress the importance of model uncertainty estimation in evaluating the impact of an event, particularly for emergency response purposes.

An aspect that we have not considered in this work is that the formulation for the Laplacian operator in eq. (13) is general and can take on various forms. To date, we have only considered a smoothing formulation but other cases are possible. In particular, one can assume no parameter correlations via $\mathbf{L}=\mathbf{I}$. Uncertainties inferred with the approach proposed here will depend on such choice but this dependence has not been explored in this article. In addition, the assumed prior covariance matrix can be a function of a number of hyperparameters. In particular, our smoothing prior depends on $\tilde{\alpha}$. Strictly speaking, $\tilde{\alpha}$ is given by a probability distribution that can be inferred (Fukuda \& Johnson 2008; Kubo et al. 2016). We use a point estimate for $\tilde{\alpha}$, based on the discrepancy principle, but note that this is a simplification and that we have currently no information about the linearization error made by using a point estimate. In the MCMC sampling, we employ for comparison (Figs 7 and 8), we do not treat $\tilde{\alpha}$ as an unknown and thus cannot quantify the linearization error due to $\tilde{\alpha}$. This requires further study since strong nonlinearities could cause poor linearized uncertainty estimates in some cases. To illustrate this point, we show in Fig. 12 slip distributions obtained with half and twice the value of $\tilde{\alpha}$ employed in the inversion. Although both models provide reasonable $\chi^{2}$ misfits, peak slips are considerably different.

However, we note that many current FFI studies do not address the issue of slip uncertainty and are typically based exclusively on point estimates to infer rupture properties and sometimes do not indicate how regularization is applied. We assert that this may be one of the reasons that discrepancies of slip estimates exist in the literature for the same event (e.g. Beresnev 2003; Minson et al. 2013). The Bayesian approach with uncertainty quantification is convenient for FFI since it provides more robust solutions to the ill-posed inverse problem. The efficiency of our method is such that 


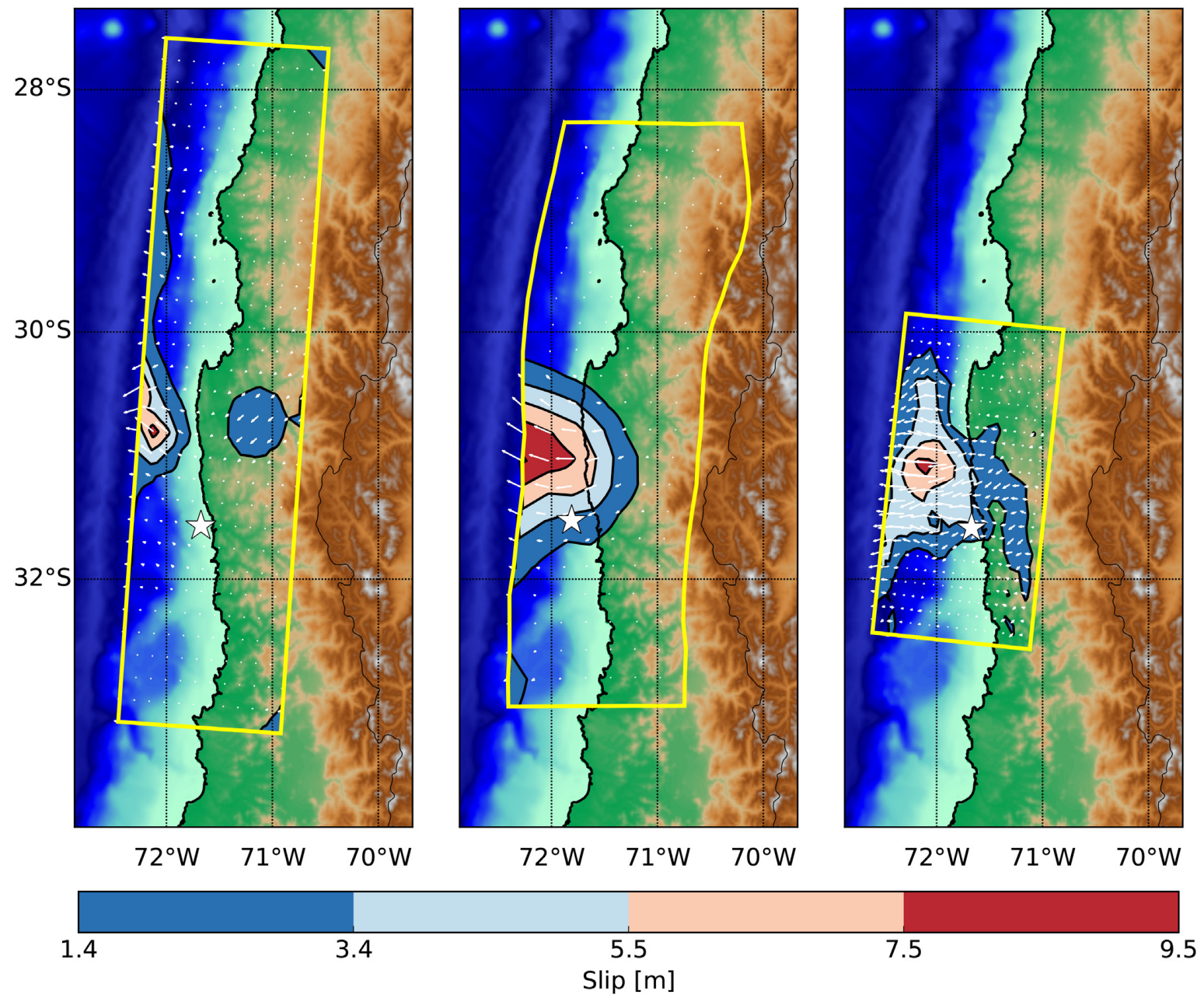

Figure 9. Comparison of slip models for the Illapel event from different inversions. Left-hand panel: Model from this study that utilizes surface waves and a $\log$-smoothing regularization scheme. The estimated magnitude is $M_{\mathrm{W}}=8.23$. Centre: Solution by Benavente et al. (2016) that inverted only long period $W$ phases and considers a standard MTWM with linear smoothing. Right-hand panel: Solution by Hayes (2015) that performs joint inversion of body and surface waves, standard linear smoothing and the nonlinear inversion method of Ji et al. (2002).
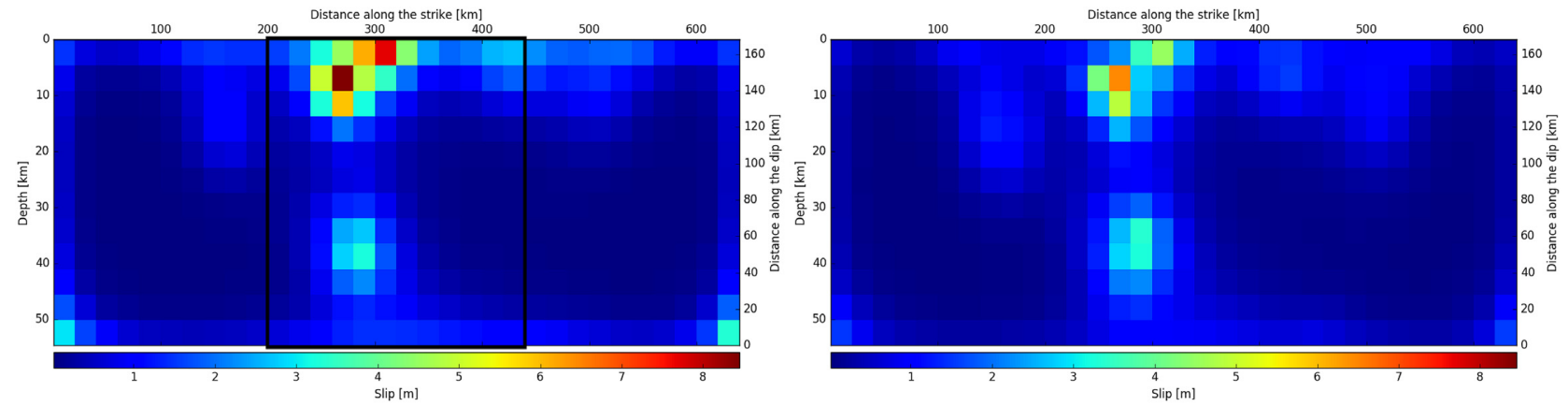

Figure 10. Mean of the cumulative slip distribution (left-hand panel) and associated 95 per cent CI (right-hand panel). Corresponding models for percentiles 2.5 and 97.5 are shown in Fig. S13 of the Supporting Information. The region enclosed by the black rectangle includes all the relevant slip features.

it can be carried out on personal computers, making it more broadly applicable in comparison to the MCMC sampling which requires high-performance computer infrastructure. This may be useful, for instance, to quickly quantify the robustness of early finite fault models that can be employed to assess the tsunamigenic potential of an event (e.g. Benavente \& Cummins 2013; Cienfuegos et al. 2018; Crowell et al. 2018). Nocquet (2018) has devised a similar inversion method that also implements positivity constraints while avoiding the MCMC sampling. Instead of using a log-normal prior in slip, Nocquet (2018) relies on a truncated Gaussian prior to realize slip positivity. As a result, while the MAP can be found using the standard NNLS algorithm, evaluation of parameter marginals as well as posterior mean and covariances require complex numerical integration over an hyper-rectangle. In contrast, the approach outlined here relies on a standard nonlinear Newton conjugate gradient algorithm, to find the MAP and then, an approximated posterior 


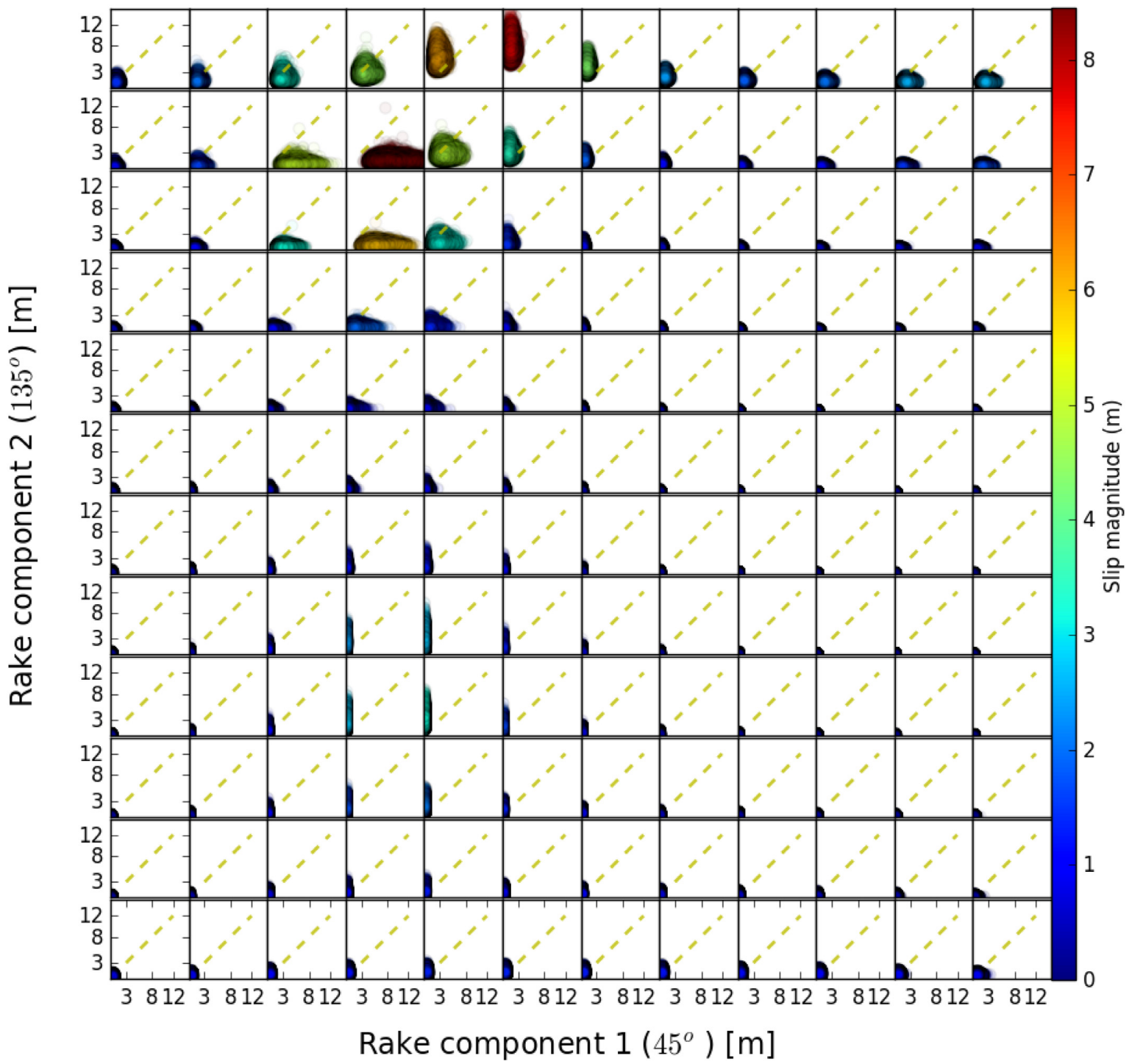

Figure 11. 2-D marginals for the two rake components of the subfaults within the region enclosed by the black rectangle in Fig. 10. Layout is the same as Fig. 10, with the top subfaults located at the shallowest portion of the fault. The dashed yellow line indicates a rake of $90^{\circ}$. For each marginal the colour indicates the mean of the slip magnitude, which is the same as in Fig. 10.
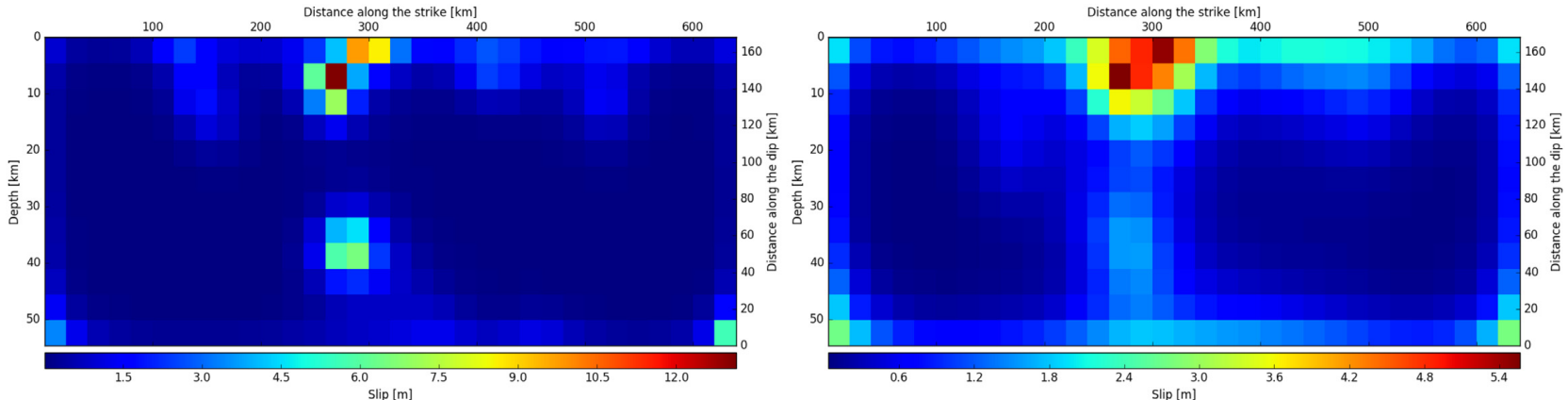

Figure 12. Mean models for cumulative slip distribution using half (left-hand panel) and twice (right-hand panel) the optimal smoothness. The former gives a $\chi^{2}$ of 84 per cent while the latter amounts to 119 per cent, both relative to the optimal $\chi^{2}$ used in the inversion.

parameter marginals, mean and covariance matrix can be evaluated analytically. We note that both methods show excellent agreement with equivalent MCMC sampling techniques.
While the algorithm was applied to surface waves records, the formulation is general and applicable for various types of data. Other teleseismic, geodetic, continuous GPS and strong motion data can also be used in a similar scheme, since the forward problem is 
also linear (or linearizable via MTWM). Although we have only focused on the use of a single data type, this approach can also be employed in joint FFI. Since we utilize a Bayesian framework, this would provide a quantitative means to evaluate the uncertainties. Since, from a Bayesian point of view, data weights can be seen as hyperparameters of the likelihood function, one could address the problem of determining the relative data weights by direct MCMC sampling of them. This approach is explored by Fukuda \& Johnson (2010) for a joint inversion of GPS, InSAR and EDM data. However, in that study a multivariate Gaussian prior for slip (linear) parameters is employed for efficiency, so that positivity constraints are not imposed. This is an interesting aspect to be explored since the impact of combining several data sets on model uncertainties has not been studied extensively, perhaps due to the high computational cost associated with previous methods.

\section{ACKNOWLEDGEMENTS}

We thank Dr. Stan E. Dosso for proving insightful comments on a preliminary version of the manuscript. We thank Dr. Chen Ji and one anonymous reviewer for their thorough review of a previous version of this article. We also appreciate the input and constructive reviews from two anonymous reviewers that improved this article. Data were acquired from the International Federation of Digital Seismograph Networks (FDSN) web service.

\section{REFER E N CES}

Akaike, H., 1974. A new look at the statistical model identification, IEEE Trans. Autom. Control, 19(6), 716-723.

Akaike, H., 1980. Likelihood and the Bayes procedure, Trabajos de estadística y de investigación operativa, 31(1), 143-166.

Amey, R., Hooper, A. \& Walters, R., 2018. A Bayesian method for incorporating self-similarity into earthquake slip inversions, J. geophys. Res., 123(7), 6052-6071.

Aránguiz, R. et al., 2016. The 16 September 2015 Chile Tsunami from the post-tsunami survey and numerical modeling perspectives, Pure appl. Geophys., 173(2),1-16.

Aster, R., Borchers, B. \& Thurber, C., 2005. Parameter Estimation and Inverse Problems, Elsevier Academic Press.

Benavente, R. \& Cummins, P.R., 2013. Simple and reliable finite fault solutions for large earthquakes using the W-phase: the Maule $\left(M_{\mathrm{W}}=8.8\right)$ and Tohoku $\left(M_{\mathrm{w}}=9.0\right)$ earthquakes, Geophys. Res. Lett., 40(14), 3591-3595.

Benavente, R., Cummins, P.R. \& Dettmer, J., 2016. Rapid automated Wphase slip inversion for the Illapel great earthquake $\left(2015, M_{\mathrm{W}}=8.3\right)$, Geophys. Res. Lett., 43(5), 1910-1917.

Beresnev, I.A., 2003. Uncertainties in finite-fault slip inversions: to what extent to believe? (a critical review), Bull. seism. Soc. Am., 93, 24452458.

Box, G.E. \& Tiao, G.C., 1968. A Bayesian approach to some outlier problems, Biometrika, 55(1), 119-129.

Cartinhour, J., 1990. One-dimensional marginal density functions of a truncated multivariate normal density function, Commun. Stat. - Theory Methods, 19(1), 197-203.

Cienfuegos, R., Catalán, P.A., Urrutia, A., Benavente, R., Aránguiz, R. \& González, G., 2018. What can we do to forecast tsunami hazards in the near field given large epistemic uncertainty in rapid seismic source inversions? Geophys. Res. Lett., 45(10), 4944-4955.

Constable, S., Parker, R. \& Constable, C., 1987. Occam's inversion: a practical algorithm for generating smooth models from electromagnetic sounding data, Geophysics, 52, 289-300.

Craven, P. \& Wahba, G., 1978. Smoothing noisy data with spline functions, Numer. Math., 31(4), 377-403.

Crowell, B.W., Melgar, D. \& Geng, J., 2018. Hypothetical real-time GNSS modeling of the $2016 M_{\mathrm{W}} 7.8$ kaikōura earthquake: perspectives from ground motion and tsunami inundation prediction, Bull. seism. Soc. Am., 108(3B), 1736-1745.

Dettmer, J., Benavente, R., Cummins, P.R. \& Sambridge, M., 2014. Transdimensional finite-fault inversion, Geophys. J. Int., 199(2), 735-751.

Dettmer, J., Dosso, S.E. \& Holland, C.W., 2007. Uncertainty estimation in seismo-acoustic reflection travel-time inversion, J. acoust. Soc. Am., 122, $161-176$.

Dettmer, J., Molnar, S., Steininger, G., Dosso, S.E. \& Cassidy, J.F., 2012. Trans-dimensional inversion of microtremor array dispersion data with hierarchical autoregressive error models, Geophys. J. Int., 188(2), 719734.

Duputel, Z., Agram, P.S., Simons, M., Minson, S.E. \& Beck, J.L., 2014. Accounting for prediction uncertainty when inferring subsurface fault slip, Geophys. J. Int., 197, 464-482.

Duputel, Z., Rivera, L., Fukahata, Y. \& Kanamori, H., 2012. Uncertainty estimations for seismic source inversions, Geophys. J. Int., 190(2), 12431256.

Duputel, Z. et al., 2015. The Iquique earthquake sequence of April 2014: Bayesian modeling accounting for prediction uncertainty, Geophys. Res. Lett., 42(19), 7949-7957.

Ekström, G., Tromp, J. \& Larson, E.W., 1997. Measurements and global models of surface wave propagation, J. geophys. Res., 102(B4), 81378157.

Fukuda, J. \& Johnson, K.M., 2008. A fully Bayesian inversion for spatial distribution of fault slip with objective smoothing, Bull. seism. Soc. Am., 98(3), 1128-1146.

Fukuda, J. \& Johnson, K.M., 2010. Mixed linear-non-linear inversion of crustal deformation data: Bayesian inference of model, weighting and regularization parameters, Geophys. J. Int., 181(3), 1441-1458.

Genz, A. \& Bretz, F., 2009. Computation of Multivariate Normal and $t$ Probabilities, Vol. 195, Springer Science \& Business Media.

Hallo, M. \& Gallovič, F., 2016. Fast and cheap approximation of green function uncertainty for waveform-based earthquake source inversions, Geophys. J. Int., 207(2), 1012-1029.

Hansen, P.C., 1992. Analysis of discrete ill-posed problems by means of the L-curve, SIAM Rev, 34(4), 561-580.

Hartzell, S. \& Heaton, T., 1983. Inversion of strong ground motion and teleseismic waveform data for the fault rupture history of the 1979 Imperial Valley, California, earthquake, Bull. seism. Soc. Am., 73(6A), 1553-1583.

Hartzell, S., Liu, P. \& Mendoza, C., 1996. The 1994 Northridge, California, earthquake: investigation of rupture velocity, risetime, and high-frequency radiation, J. geophys. Res., 101(B9), 20 091-20 108.

Hayes, G., 2015. Preliminary Finite Fault Results for the Sep 16, $2015 M_{w}$ 8.2. Available at: https://earthquake.usgs.gov/earthquakes/eventpage/us 20003k7a\#finite-fault(a ccessed: 2018 June 1).

Ide, S., 2007. Slip Inversion, in Treatise on Geophysics, Vol. 4, ed. Kanamori, H., Elsevier.

Ide, S., Takeo, M. \& Yoshida, Y., 1996. Source process of the 1995 Kobe earthquake: determination of spatio-temporal slip distribution by Bayesian modeling, Bull. seism. Soc. Am., 86(3), 547-566.

Ji, C., Wald, D. \& Helmberger, D., 2002. Source description of the 1999 Hector Mine, California, earthquake, part I: wavelet domain inversion theory and resolution analysis, Bull. seism. Soc. Am., 92(4), 1192-1207.

Jones, E., et al., 2001. SciPy: Open source scientific tools for Python (online; accessed 2016 February 20).

Kanamori, H. \& Stewart, G.S., 1976. Mode of the strain release along the gibbs fracture zone, mid-atlantic ridge, Phys. Earth planet. Inter., 11(4), 312-332.

Kubo, H., Asano, K., Iwata, T. \& Aoi, S., 2016. Development of fully Bayesian multiple-time-window source inversion, Geophys. J. Int., 204(3), 1601-1619.

Lawson, C.L. \& Hanson, R.J., 1974. Solving Least Squares Problems, vol. 161, SIAM.

Lindley, D.V. \& El-Sayyad, G., 1968. The Bayesian estimation of a linear functional relationships, J. Royal Stat. Soc. B, 30, 190-202.

Lindley, D.V. \& Smith, A.F., 1972. Bayes estimates for the linear model, J. Royal Stat. Soc. B, 34, 1-41. 
MacKay, D.J., 2003. Information Theory, Inference and Learning Algorithms, Cambridge Univ. Press.

Melgar, D. et al., 2016. Slip segmentation and slow rupture to the trench during the 2015, $M_{\mathrm{w}} 8.3$ Illapel, Chile earthquake, Geophys. Res. Lett, 43, 961-966.

Minson, S., Murray, J.R., Langbein, J.O. \& Gomberg, J.S., 2014a. Real-time inversions for finite fault slip models and rupture geometry based on high-rate GPS data, J. geophys. Res., 119(4), 3201-3231.

Minson, S., Simons, M. \& Beck, J., 2013. Bayesian inversion for finite fault earthquake source models I-theory and algorithm, Geophys. J. Int., 194(3), 1701-1726.

Minson, S. et al., 2014b. Bayesian inversion for finite fault earthquake source models-II: the 2011 great Tohoku-oki, Japan earthquake, Geophys. J. Int., 198(2), 922-940.

Monelli, D. \& Mai, P., 2008. Bayesian inference of kinematic earthquake rupture parameters through fitting of strong motion data, Geophys. J. Int., 173(1), 220-232.

Nocquet, J., 2018. Stochastic static fault slip inversion from geodetic data with non-negativity and bound constraints, Geophys. J. Int., 214(1), 366385

Olson, A.H. \& Apsel, R.J., 1982. Finite faults and inverse theory with applications to the 1979 Imperial Valley earthquake, Bull. seism. Soc. Am., 72(6A), 1969-2001.

Press, W., Teukolsky, S., Vetterling, W. \& Flannery, B., 1992. Numerical Recipes in Fortran 77: The Art of Scientific Computing, Cambridge Univ. Press.

Rapid Finite Fault Inversion for Megathrust Earthquakes, 2016. PhD Thesis, Australian National University, https://openresearch-repository.anu.edu. au/handle/1885/108615.

Schwarz, G. et al., 1978. Estimating the dimension of a model, Ann. Stat., 6(2), 461-464.

Sivia, D. \& Skilling, J., 2006. Data Analysis: A Bayesian Tutorial, Oxford University Press.

Smith, A.F., 1973. A general bayesian linear model, J. Royal Stat. Soc. B, $67-75$.

Tarantola, A., 2005. Inverse Problem Theory and Methods for Model Parameter Estimation, SIAM.

Tarantola, A. \& Valette, B., 1982. Inverse problems = quest for information, J. Geophys., 50, 159-170.

Tikhonov, A.N. \& Arsenin, V.Y., 1977. Solutions of Ill-Posed Problems, Winston and Sons.

Tilmann, F. et al., 2016. The 2015 Illapel earthquake, central Chile: a type case for a characteristic earthquake? Geophys. Res. Lett., 43, 574-583.

Towards rapid uncertainty estimation in linear finite fault inversion with positivity constraints, 2015. https://agu.confex.com/agu/fm15/webprogram/P aper76601.html, http://adsabs.harvard.edu/abs/2015AGUFM.S54A..06.

Wright, S. \& Nocedal, J., 1999. Numerical Optimization, Vol. 35, Springer.

Yabuki, T. \& Matsu'ura, M., 1992. Geodetic data inversion using a Bayesian information criterion for spatial distribution of fault slip, Geophys. J. Int., 109(2), 363-375.

Yagi, Y. \& Fukahata, Y., 2011. Introduction of uncertainty of Green's function into waveform inversion for seismic source processes, Geophys. J. Int., 186(2), 711-720.

Ye, L., Lay, T., Kanamori, H. \& Koper, K.D., 2015. Rapidly estimated seismic source parameters for the 16 September 2015 Illapel, Chile $M_{\mathrm{w}}$ 8.3 earthquake, Pure appl. Geophys., 173, 321-332.

Yoshida, S., 1989. Waveform inversion using ABIC for the rupture process of the 1983 Hindu Kush earthquake, Phys. Earth planet. Inter., 56(3), 389-405.

\section{SUPPORTING INFORMATION}

Supplementary data are available at $G J I$ online.

Figure S1. Models for percentiles 2.5 (left-hand panel) and 97.5 (right-hand panel) for the simulation described in Section 3. Noise level is given by $\gamma=2$ per cent.
Figure S2. Models for percentiles 2.5 (left-hand panel) and 97.5 (right-hand panel) for the simulation described in Section 3. Noise level is given by $\gamma=5$ per cent.

Figure S3. Models for percentiles 2.5 (left-hand panel) and 97.5 (right-hand panel) for the simulation described in Section 3 with a large azimuthal gap. Noise level is given by $\gamma=5$ per cent.

Figure S4. Data prediction from our approximated posterior for the Illapel event. The blue lines are the measured data and the yellow lines are predictions for 1000 samples drawn from the posterior PDF. For each subplot, station name and component (T: Transverse, $\mathrm{Z}$ : Up) are displayed in the top-right corner.

Figure S5. Data prediction from our approximated posterior for the Illapel event. The blue lines are the measured data and the yellow lines are predictions for 1000 samples drawn from the posterior PDF. For each subplot, station name and component (T: Transverse, $\mathrm{Z}$ : Up) are displayed in the top-right corner.

Figure S6. Data prediction from our approximated posterior for the Illapel event. The blue lines are the measured data and the yellow lines are predictions for 1000 samples drawn from the posterior PDF. For each subplot, station name and component (T: Transverse, $\mathrm{Z}$ : Up) are displayed in the top-right corner.

Figure S7. PDFs for each slip parameter in our inversion for the Illapel event. Here we show the marginals for the rake component of $45^{\circ}$ and the first time window. Plots for the rest of the parameters can be found in Figs S8-S12. The layout is the same as in Fig. 8 so that the first row corresponds to the shallowest subfaults and first column to the southernmost subfaults. The red lines indicate the marginal obtained by our approximated analytic posterior and the blue histograms show the results from an MCMC sampling of the full posterior.

Figure S8. PDFs for each slip parameter in our inversion for the Illapel event. Marginals are shown for the rake component of $45^{\circ}$ and the second time window. Legends and layout are the same as in Fig. S7.

Figure S9. PDFs for each slip parameter in our inversion for the Illapel event. Marginals are shown for the rake component of $45^{\circ}$ and the third time window. Legends and layout are the same as in Fig. S7.

Figure S10. PDFs for each slip parameter in our inversion for the Illapel event. Marginals are shown for the rake component of $135^{\circ}$ and the first time window. Legends and layout are the same as in Fig. S7.

Figure S11. PDFs for each slip parameter in our inversion for the Illapel event. Marginals are shown for the rake component of $135^{\circ}$ and the second time window. Legends and layout are the same as in Fig. S7.

Figure S12. PDFs for each slip parameter in our inversion for the Illapel event. Marginals are shown for the rake component of $135^{\circ}$ and the third time window. Legends and layout are the same as in Fig. S7.

Figure S13. Models for percentiles 2.5 (left-hand panel) and 97.5 (right-hand panel) for the inversion based on observed surface wave data described in Section 4.

Please note: Oxford University Press is not responsible for the content or functionality of any supporting materials supplied by the authors. Any queries (other than missing material) should be directed to the corresponding author for the paper. 


\section{APPENDIX A: HESSIAN AND \\ GRA D I E N T}

In this appendix we derive the expressions for the gradient and Hessian of the objective function eq. (16) which is expressed as

$\tilde{\psi}(\mathbf{s})=(\mathbf{G e}(\mathbf{s})-\mathbf{d})^{T} \mathbf{C}_{\mathbf{d}}{ }^{-1}(\mathbf{G e}(\mathbf{s})-\mathbf{d})+\tilde{\alpha}^{-2}(\mathbf{L s})^{T}(\mathbf{L s})$.

Introducing the Cholesky decomposition of $\mathbf{C}_{\mathbf{d}}$,

$\mathbf{C}_{\mathbf{d}}{ }^{-1}=\left(\mathbf{K K}^{T}\right)^{-1}=\mathbf{K}^{-T} \mathbf{K}^{-1}$,

where $\mathbf{K}$ is a lower triangular matrix and $\mathbf{K}^{-T}=\left(\mathbf{K}^{-1}\right)^{T}$, we can rewrite eq. (A1) as

$\tilde{\psi}(\mathbf{s})=(\tilde{\mathbf{G}} \mathbf{e}(\mathbf{s})-\tilde{\mathbf{d}})^{T}(\tilde{\mathbf{G}} \mathbf{e}(\mathbf{s})-\tilde{\mathbf{d}})+\tilde{\alpha}^{-2}(\mathbf{L s})^{T}(\mathbf{L s})$,

where

$\tilde{\mathbf{G}}=\mathbf{K}^{-1} \mathbf{G}, \quad \tilde{\mathbf{d}}=\mathbf{K}^{-1} \mathbf{d}$.

For convenience we name the first and second terms of eq. (A3) $\tilde{\psi}(\mathbf{s})^{(I)}$ and $\tilde{\psi}(\mathbf{s})^{(I I)}$, respectively, so that

$\tilde{\psi}(\mathbf{s})=\tilde{\psi}^{(I)}(\mathbf{s})+\tilde{\psi}^{(I I)}(\mathbf{s})$.

By applying index notation and rearranging, we find that

$$
\begin{aligned}
\frac{\partial \tilde{\psi}^{(I I)}(\mathbf{s})}{\partial s_{l}} & =\alpha^{-2} \frac{\partial}{\partial s_{l}} \sum_{i}\left(\sum_{j} L_{i j} s_{j}\right)^{2} \\
& =2 \alpha^{-2} \sum_{i}\left(\sum_{j} L_{i j} s_{j}\right)\left(\sum_{m} L_{i m} \delta_{l m}\right) \\
& =2 \alpha^{-2} \sum_{i}\left(\sum_{j} L_{i j} s_{j}\right) L_{i l} \\
& =2 \alpha^{-2}\left(\mathbf{L}^{T} \mathbf{L s}\right)_{l},
\end{aligned}
$$

where $\delta_{i j}$ is the Kronecker delta. From this result, it can be seen that

$\frac{\partial \tilde{\psi}^{(I I)}(\mathbf{s})}{\partial s_{k} \partial s_{l}}=2 \alpha^{-2}\left(\mathbf{L}^{T} \mathbf{L}\right)_{l k}$.
The derivatives of $\tilde{\psi}^{(I)}(\mathbf{s})$ can be obtained similarly:

$$
\begin{aligned}
\frac{\partial \tilde{\psi}^{(I)}(\mathbf{s})}{\partial s_{l}} & =\frac{\partial}{\partial s_{l}} \sum_{i}\left(\sum_{j} \tilde{G}_{i j} e_{j}-\tilde{d}_{i}\right)^{2} \\
& =2 \sum_{i}\left(\sum_{j} \tilde{G}_{i j} e_{j}-\tilde{d}_{i}\right) \frac{\partial}{\partial s_{l}}\left(\sum_{k} \tilde{G}_{i k} e_{k}-\tilde{d}_{k}\right) \\
& =2 \sum_{i}\left(\sum_{j} \tilde{G}_{i j} e_{j}-\tilde{d}_{i}\right)\left(\sum_{k} \tilde{G}_{i k} e_{k} \delta_{k l}\right) \\
& =2 e_{l} \sum_{i} \tilde{G}_{i l} \sum_{j}\left(\tilde{G}_{i j} e_{j}-\tilde{d}_{i}\right) \\
& =2\left[\mathbf{e} \otimes \mathbf{G}^{T} \mathbf{C}_{\mathbf{d}}{ }^{-1}(\mathbf{G e}-\mathbf{d})\right]_{l} \\
\text { and } &
\end{aligned}
$$

$$
\begin{aligned}
\frac{\partial \tilde{\psi}^{(I I)}(\mathbf{s})}{\partial s_{k} \partial s_{l}} & =2 e_{l} \frac{\partial}{\partial s_{k}}\left[\sum_{i} \tilde{G}_{i l} \sum_{j}\left(\tilde{G}_{i j} e_{j}-\tilde{d}_{i}\right)\right] \\
& +2 \frac{\partial e_{l}}{\partial s_{k}} \sum_{i} \tilde{G}_{i l} \sum_{j}\left(\tilde{G}_{i j} e_{j}-\tilde{d}_{i}\right) \\
& =2 e_{l} \sum_{i} \tilde{G}_{i l} \sum_{j} \tilde{G}_{i j} e_{j} \delta_{j k} \\
& +2 e_{l} \delta_{k l} \sum_{i} \tilde{G}_{i l} \sum_{j}\left(\tilde{G}_{i j} e_{j}-\tilde{d}_{i}\right) \\
& =2 e_{l} e_{k} \sum_{i} \tilde{G}_{i l} \tilde{G}_{i k} \\
& +2 e_{l} \delta_{k l} \sum_{i} \tilde{G}_{i l} \sum_{j}\left(\tilde{G}_{i j} e_{j}-\tilde{d}_{i}\right) \\
& =2\left\{\mathbf{e e}^{T} \otimes \mathbf{G}^{T} \mathbf{C}_{\mathbf{d}}{ }^{-1} \mathbf{G}\right. \\
& \left.+\operatorname{diag}\left[\mathbf{e} \otimes \mathbf{G}^{T} \mathbf{C}_{\mathbf{d}}{ }^{-1}(\mathbf{G e}-\mathbf{d})\right]\right\}_{k l} .
\end{aligned}
$$

Taking the derivative of eq. (A5) and using eqs (A8) and (A6) we obtain

$\nabla \psi(\mathbf{s})=2 \mathbf{e} \otimes \mathbf{G}^{T} \mathbf{C}_{\mathbf{d}}{ }^{-1}(\mathbf{G e}-\mathbf{d})+2 \alpha^{-2} \mathbf{L}^{T} \mathbf{L s}$,

which is the same as eq. (20). Likewise, taking the second-order derivatives of eq. (A5) and using eqs (A9) and (A7) yields

$$
\begin{aligned}
\nabla \nabla \psi(\mathbf{s})= & 2 \mathbf{e e}^{T} \otimes \mathbf{G}^{T} \mathbf{C}_{\mathbf{d}}{ }^{-1} \mathbf{G} \\
& +2 \operatorname{diag}\left[\mathbf{e} \otimes \mathbf{G}^{T} \mathbf{C}_{\mathbf{d}}{ }^{-1}(\mathbf{G e}-\mathbf{d})\right] \\
& +2 \alpha^{-2} \mathbf{L}^{T} \mathbf{L},
\end{aligned}
$$

which is the same as eq. (21). 